\title{
Review \\ Objectively Measured Physical Activity, Sedentary Behavior and Functional Performance before and after Lower Limb Joint Arthroplasty: A Systematic Review with Meta-Analysis
}

\author{
Matic Sašek ${ }^{1,2}$, Žiga Kozinc ${ }^{1,3}\left(\mathbb{D}\right.$, Stefan Löfler ${ }^{4,5,6}\left(\mathbb{D}\right.$, Christian Hofer ${ }^{6}$ and Nejc Šarabon ${ }^{1,2,3,7, *(\mathbb{D})}$ \\ 1 Faculty of Health Sciences, University of Primorska, Polje 42, SI-6310 Izola, Slovenia; \\ matic.sasek@fvz.upr.si (M.S.); ziga.kozinc@fvz.upr.si (Ž.K.) \\ 2 InnoRenew CoE, Livade 6, SI-6310 Izola, Slovenia \\ 3 Andrej Marušič Institute, University of Primorska, Muzejski trg 2, SI-6000 Koper, Slovenia \\ 4 Physiko- \& Rheumatherapie, Institute for Physical Medicine and Rehabilitation, 3100 St. Pölten, Austria; \\ Stefan.Loefler@rehabilitation.lbg.ac.at \\ 5 Centre of Active Ageing, Competence Centre for Health, Prevention and Active Ageing, \\ 3100 St. Pölten, Austria \\ 6 Ludwig Boltzmann Institute for Rehabilitation Research, Neugebäudeplatz 1, 3100 St. Pölten, Austria; \\ Christian.Hofer@rehabilitation.lbg.ac.at \\ 7 Laboratory for Motor Control and Motor Behavior, S2P, Science to Practice, Ltd., Tehnološki Park 19, \\ SI-1000 Ljubljana, Slovenia \\ * Correspondence: nejc.sarabon@fvz.upr.si
}

check for updates

Citation: Sašek, M.; Kozinc, Ž.; Löfler, S.; Hofer, C.; Šarabon, N. Objectively Measured Physical Activity, Sedentary Behavior and Functional Performance before and after Lower Limb Joint Arthroplasty: A Systematic Review with MetaAnalysis. J. Clin. Med. 2021, 10, 5885. https://doi.org/10.3390/jcm10245885

Academic Editors: Zan Gao and Jung Eun Lee

Received: 14 November 2021 Accepted: 13 December 2021 Published: 15 December 2021

Publisher's Note: MDPI stays neutral with regard to jurisdictional claims in published maps and institutional affiliations.

Copyright: (c) 2021 by the authors. Licensee MDPI, Basel, Switzerland. This article is an open access article distributed under the terms and conditions of the Creative Commons Attribution (CC BY) license (https:// creativecommons.org/licenses/by/ $4.0 /)$.

\begin{abstract}
Patients after joint arthroplasty tend to be less physically active; however, studies measuring objective physical activity (PA) and sedentary behavior (SB) in these patients provide conflicting results. The aim of this meta-analysis was to assess objectively measured PA, SB and performance at periods up to and greater than 12 months after lower limb arthroplasty. Two electronic databases (PubMed and Medline) were searched to identify prospective and cross-sectional studies from 1 January 2000 to 31 December 2020. Studies including objectively measured SB, PA or specific performance tests in patients with knee or hip arthroplasty, were included in the analyses both preand post-operatively. The risk of bias was assessed using the Scottish Intercollegiate Guidelines Network (SIGN). After identification and exclusion, 35 studies were included. The data were analyzed using the inverse variance method with the random effects model and expressed as standardized mean difference and corresponding 95\% confidence intervals. In total, we assessed 1943 subjects with a mean age of 64.9 ( \pm 5.85$)$. Less than 3 months post-operative, studies showed no differences in PA, SB and performance. At 3 months post-operation, there was a significant increase in the 6 min walk test (6MWT) (SMD 0.65; CI: 0.48, 0.82). After 6 months, changes in moderate to vigorous physical activity (MVPA) (SMD 0.33; CI: 0.20, 0.46) and the number of steps (SMD 0.45; CI: 0.34, 0.54) with a large decrease in the timed-up-and-go test (SMD -0.61; CI: $-0.94,-0.28)$ and increase in the 6MWT (SMD 0.62; CI: 0.26-0.98) were observed. Finally, a large increase in MVPA (SMD 0.70; CI: 0.53-0.87) and a moderate increase in step count (SMD 0.52; CI: 0.36, 0.69) were observed after 12 months. The comparison between patients and healthy individuals pre-operatively showed a very large difference in the number of steps (SMD -1.02; CI: $-1.42,-0.62$ ), but not at 12 months (SMD $-0.75 ;-1.89$, 0.38). Three to six months after knee or hip arthroplasty, functional performance already exceeded pre-operative levels, yet PA levels from this time period remained the same. Although PA and functional performance seemed to fully restore and exceed the pre-operation levels at six to nine months, SB did not. Moreover, PA remained lower compared to healthy individuals even longer than twelve months post-operation. Novel rehabilitation protocols and studies should focus on the effects of long-term behavioral changes (increasing PA and reducing SB) as soon as functional performance is restored.
\end{abstract}

Keywords: surgery; sitting; lower limb; rehabilitation; orthopedics; replacement 


\section{Introduction}

Osteoarthritis (OA) is the most prevalent degenerative disease of the musculoskeletal system [1]. In many cases, especially in older adults, it causes joint pain and limits functional ability. When the joints of the lower limbs are affected, weight-bearing activities, such as walking or kneeling, can be severely impaired [2]. Because severe OA of the lower limb joints drastically affects quality of life, hip and knee arthroplasty are being considered as viable treatment options when conservative treatment does not lead to the relief of symptoms. The increasing prevalence of $\mathrm{OA}$ associated with ageing of the population is reflected in a proportionally higher number of total joint arthroplasty procedures. Consequently, total hip and knee arthroplasties are among the most common elective surgical procedures worldwide [3,4]. The commonly expected outcomes of surgery are pain relief, increased mobility, function and higher quality of life, which are associated with increased physical activity (PA) levels and sport participation [5-7].

Sedentary behavior (SB) is one of the major risk factors for developing the chronic non-communicable disease. Together, these diseases are estimated to cause 71 percent of all deaths worldwide [8]. Therefore, reducing SB is useful for improving longevity, long-term health and well-being. It is even better to replace SB with PA, which is highly effective in preventing and treating diseases $[9,10]$. According to the latest guidelines, $150-300 \mathrm{~min}$ of moderate to vigorous intensity or 75-150 min of vigorous intensity PA per week is considered sufficient to maintain health and well-being [11]. Because of its physiological benefits, PA is a part of rehabilitation for a variety of disabilities, including osteoarthritis and joint arthroplasty. The dose-response relationship between levels of PA and health in middle-aged and older adults is strong [12]. The positive effects of physical activity on health are well established in practice and have a strong theoretical background. Finally, the cost-effectiveness of physical activity from the perspective of an individual's health and social and economic well-being is undisputed [13,14].

Over the last two decades, the development of high-technology measurement tools has enabled the quantification of PA and SB levels. Compared to questionnaires, newgeneration accelerometers provide a more reliable and objective insight into an individual's physical activity profile [15]. Many systematic reviews and meta-analyses have examined objectively measured and self-reported PA levels or sport participation in patients after lower limb arthroplasty [7,16-19]. Notably, physical function is shown to recover to approximately $80 \%$ of that of controls [20]. To our knowledge, only one systematic review considered objectively measured SB after TKA and provided heterogenous results. The authors suggested that knowing SB post-operative trajectories in detail would help in tailoring targeted interventions [16]. Although total joint arthroplasty increases functional capacity and relieves OA-related pain, it remains unclear whether and to what extent patients change their objectively measured PA, SB and functional performance post-operation $[17,21]$.

For this purpose, the primary aim of this paper was to review the studies that prospectively investigated objectively measured $\mathrm{PA}, \mathrm{SB}$ and functional performance after lower limb arthroplasty for up to 12 months. The secondary aim of the study was to pre- and post-operatively compare the OA patients with healthy peers. We hypothesized that (a) patient groups will exhibit lower PA, higher SB and lower functional performance compared to healthy peers and (b) these outcomes will improve throughout the first 12 months following the operation. While some systematic reviews regarding this topic already exist, the number of studies reporting objectively measured PA and SB have been increasing in the last years. Numerous studies published enabled us to provide objectively measured PA and SB change trajectories. By including functional performance changes, a coherent interpretation off all three determinants together could be of high value for future clinical research and rehabilitation interventions of patients after lower limb arthroplasty in accordance with the Global Physical Activity Action Plan [22]. The review was performed according to the PRISMA 2020 statement for reporting systematic reviews [23]. 


\section{Materials and Methods}

\subsection{Search Strategy}

The study protocol was conducted in accordance with the Cochrane Collaboration guidelines [24]. Two electronic databases, including PubMed and Medline, were searched from 1 January 2000 to 31 December 2020. The literature search was conducted using the keywords and terms used in the search strategy, including: "physical*" "active*" OR "sedentary" OR "sitting" AND "endoprosthesis" OR "replacement" OR "arthroplasty" OR "arthrosis" OR "arthritis" OR "osteoarthrosis" OR "osteoarthritis" AND "knee" OR "hip" OR "ankle". One author searched the databases for the consistency of search hits. In addition, the reference lists of all included articles were searched for relevant studies that were not covered by the constructed search strategy.

\subsection{Eligibility Criteria}

Studies were included if they met the following inclusion criteria: (a) the study design was longitudinal or cross-sectional and included pre-operative and at least 3 months of post-operative data, (b) the study reported on objectively measured PA or SB or measured functional performance by using the 6 Minute Walk Test (6MWT) or Time Up and Go (TUG) and (c) included adult participants with a diagnosis of knee or hip OA who underwent primary or recurrent unilateral or bilateral total or complementary joint arthroplasty. For PA, only objective methods (uni-, bi- or triaxial accelerometers or pedometers) were considered. For SB, only accelerometers were accepted as an objective method of assessment. Reviews were not included in the systematic review, but the reference list of reviews found was examined, and all studies that met the inclusion criteria were included. Studies were excluded from the review if they (a) measured PA or SB using subjective questionnaires or performance level with other tests or (b) the results were obtained only for a single timeframe (i.e., only 12 months post-operation).

\subsection{Study Selection and Quality Assessment}

The abstracts and titles of the articles were screened for eligibility by two reviewers. The full text was than obtained and reviewed by two reviewers for articles with limited eligibility and potentially eligible articles. The articles whose eligibility criteria were accepted by both reviewers after the full text review were included in the review. Any ambiguities or conflicts were resolved through discussion. To detect bias and assess methodological quality, the Scottish Intercollegiate Guidelines Network (SIGN) methodology for cohort studies was used (Table S1). One author reviewed the quality of the studies and graded them as high (++; major of criteria met), acceptable (+; most criteria met) or low (0; most criteria not met) [25].

\subsection{Data Items}

For data extraction, a prepared data sheet was used by one reviewer and checked for accuracy and consistency by a second reviewer. For all included articles, the first author, year of publication, study design, type and location of surgical procedure (knee arthroplasty (KA) or hip arthroplasty (HA) and type of procedure), participant characteristics (age, body mass index and gender distribution), type of accelerometers and outcomes were extracted and summarized in tables in sequential order. For outcomes PA, SB and functional performance, data were collected in chronological order at one pre-operative and four post-operative time periods. Means, standard deviations and sample sizes were extracted from included studies. For articles in which results were reported in medians, interquartile ranges and confidence intervals, means and standard deviations were calculated using the methods of Wan [26] and Moher [27].

\subsection{Data Analysis}

Meta-analyses were performed using Review Manager software (version 5.3, Cochrane Collaboration, London, UK). The inverse variance method with the random effects model 
was used. Differences (between patients and controls or between time points) were expressed as standardized mean differences (SMDs) and corresponding 95\% confidence intervals (Cis). When possible, mean differences were also calculated in units of measurement. Statistical heterogeneity between studies was assessed by calculating the $\mathrm{I}^{2}$ statistic. According to the Cochrane guidelines, $\mathrm{I}^{2}$ statistics of $0 \%$ to $40 \%$ may not be important, whereas $30 \%$ to $60 \%$ represent moderate heterogeneity, $50 \%$ to $90 \%$ represent substantial heterogeneity and $75 \%$ to $100 \%$ represent substantial heterogeneity. Sensitivity analysis was performed by eliminating studies one-by-one and checking whether the statistical significance of the pooled effect was affected.

\section{Results}

The initial search yielded 6059 results in the databases. Thirty-four studies met the inclusion criteria and were included in the review (Table 1). The number of steps was reported for 919 subjects (689 female and 230 male) with a mean age of $63.7( \pm 7.5)$ and 29.1 (3.7) BMI; MVPA for 358 subjects (266 female and 92 male) with a mean age of 67.9 (4.9) and 27.1 (2.7) BMI; SB for 230 subjects with a mean age of 66.5 (3.6) and 29.7 (2.9) BMI; 6MWT for a mean age of 63.7 (3.2) and 30 (2.4); and TUG for 192 subjects with average age 65.1 (2.9) and 28.6 (2.9) BMI. The flowchart of the selection process according to the PRISMA statement [27] is shown in Figure 1. Twenty-seven studies were classified as acceptable $(+)$, seven studies were classified as high quality $(++)$ and only one study was classified as low quality (0) (Table S1).

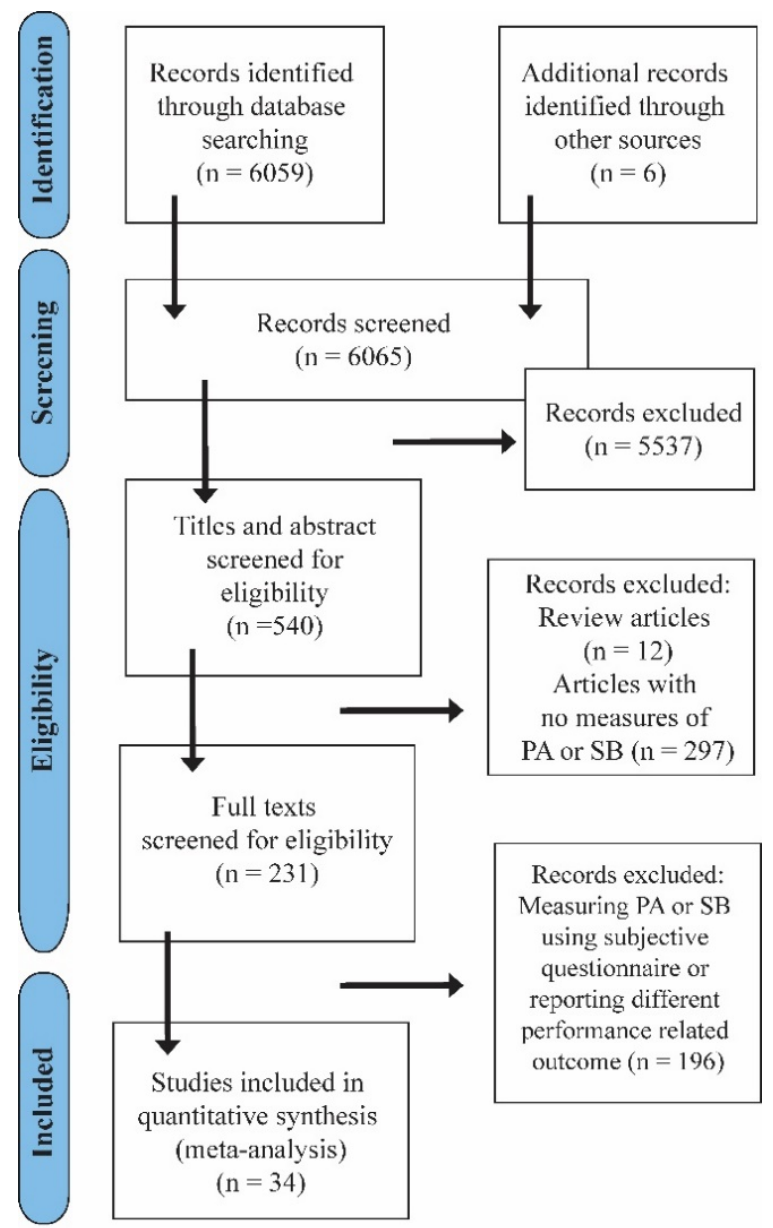

Figure 1. Summary of study search protocol. PA—physical activity; SB—sedentary behavior. 
Table 1. Summary of included studies objectively measuring physical activity, sedentary behavior and performance pre and post lower limb arthroplasty.

\begin{tabular}{|c|c|c|c|c|c|c|}
\hline Study & Study Design & $\begin{array}{c}\text { Pre-Operative } \\
\text { Descriptive Statistics }\end{array}$ & $\begin{array}{c}\text { Type and Location of } \\
\text { Arthroplasty }\end{array}$ & Outcome & Measurement & Main Conclusions \\
\hline Bade 2014 [28] & $\begin{array}{c}\text { Prospective } \\
\text { observational study }\end{array}$ & $\begin{array}{c}\mathrm{n}=64 \\
\text { sex }=50 \% \mathrm{~F}, 50 \% \mathrm{M} \\
\text { age }=64.6 \pm 8.5 \\
\mathrm{BMI}=30.6 \pm 4.8\end{array}$ & $\begin{array}{l}\text { Tricompartmental, } \\
\text { cemented TKA with } \\
\text { the medial } \\
\text { parapatellar approach. }\end{array}$ & $\begin{array}{l}\text { Performance: } \\
6 \mathrm{MWT}, \mathrm{TUG}\end{array}$ & $\begin{array}{l}\text { Pre: } 1-2 \text { weeks } \\
\text { Post: } 6 \text { months }\end{array}$ & $\begin{array}{l}\text { Acute post-operative TUG } \\
\text { performance can be used for } \\
\text { establishing a prognosis. }\end{array}$ \\
\hline Brandes 2011 [29] & $\begin{array}{c}\text { Prospective } \\
\text { observational study }\end{array}$ & $\begin{array}{c}\mathrm{n}=53 \\
\text { sex }=63 \% \mathrm{~F} ; 27 \% \mathrm{M} \\
\text { age }=65.8 \pm 5.8 \\
\mathrm{BMI}=30.7 \pm 4.1\end{array}$ & $\begin{array}{c}\text { Cemented and } \\
\text { uncemented TKA. }\end{array}$ & $\begin{array}{l}\text { SB and PA: tri-axial } \\
\text { DynaPort Activity } \\
\text { Monutor (McRoberts); } \\
\text { Step activity monitor } \\
\text { (OrhoCare Innovations) }\end{array}$ & $\begin{array}{c}\text { Pre: } 1-2 \text { weeks } \\
\text { Post: } 2 \text { months, } \\
6 \text { months, } 12 \text { months }\end{array}$ & $\begin{array}{l}\text { The activity level post treatment } \\
\text { seems to be influenced by } \\
\text { physical activity behavior prior } \\
\text { to surgery rather than by the } \\
\text { treatment itself. }\end{array}$ \\
\hline Caliskan 2020 [30] & $\begin{array}{c}\text { Prospective } \\
\text { observational study }\end{array}$ & $\begin{array}{c}\mathrm{n}=36 \\
\text { sex }=86 \% \mathrm{~F} ; 14 \% \mathrm{M} \\
\text { age }=67.3 \pm 7.7 \\
\mathrm{BMI}=33.2 \pm 5.9\end{array}$ & Primary TKA. & $\begin{array}{l}\text { SB and PA: ActiCal } \\
\text { (Philips Respironics) }\end{array}$ & $\begin{array}{l}\text { Pre: } 1 \text { week } \\
\text { Post: } 6 \text { months }\end{array}$ & $\begin{array}{c}\text { No change in sedentary } \\
\text { behavior time, increased light } \\
\text { physical activity and moderate } \\
\text { to vigorous activity. }\end{array}$ \\
\hline Casazza 2019 [31] & $\begin{array}{l}\text { Prospective } \\
\text { control study }\end{array}$ & $\begin{array}{c}\mathrm{n}=7 \\
\text { sex }=5 \mathrm{~F} ; 2 \mathrm{M} \\
\text { age }=55.6 \pm 3.5 \\
\mathrm{BMI}=32.8 \pm 1.5\end{array}$ & Primary TKA. & $\begin{array}{l}\text { SB and PA: Sensewear } \\
\text { Pro } 3 \text { (BodyMedia) }\end{array}$ & & $\begin{array}{l}\text { Non-significant trends towards } \\
\text { improved CDV fitness and } \\
\text { activity levels } 12 \text { months } \\
\text { after surgery. }\end{array}$ \\
\hline Daugaard 2018 [33] & Cross-section study & $\begin{array}{c}\mathrm{n}=52 \\
\text { sex }=50 \% \mathrm{~F} ; 50 \% \mathrm{M} \\
\text { age }=62 \pm 9.6 \\
\mathrm{BMI}=\mathrm{N} / \mathrm{A}\end{array}$ & $\begin{array}{l}\text { Primary unilateral total } \\
\text { or unicompartmental } \\
\text { knee replacement } \\
\text { surgery. }\end{array}$ & $\begin{array}{l}\text { SB and PA: tri-axial } \\
\text { accelerometer X16-mini } \\
\text { (GCdataconcepts) and } \\
\text { AX3 (Axivity) }\end{array}$ & Post: at least 5 years & $\begin{array}{l}\text { Knee OA and treatment with } \\
\text { joint replacement hardly affect } \\
\text { health-related general activity } \\
\text { but affects specific behavior. }\end{array}$ \\
\hline Dayton 2016 [34] & $\begin{array}{c}\text { Prospective } \\
\text { observational study }\end{array}$ & $\begin{array}{c}\mathrm{n}=23 \\
\text { sex }=16 \mathrm{~F} ; 7 \mathrm{M} \\
\text { age }=61.4 \pm 8.3 \\
\mathrm{BMI}=29.2 \pm 5.1\end{array}$ & $\begin{array}{l}\text { Primary unilateral } \\
\text { posterior approach THA } \\
\text { due to OA. Between June } \\
2010 \text { and August } 2011 .\end{array}$ & $\begin{array}{l}\text { Performance: } \\
6 \text { MVT, TUG }\end{array}$ & $\begin{array}{l}\text { Pre: } 2 \text { weeks } \\
\text { Post: } 1 \text { month, } \\
\quad 6 \text { months }\end{array}$ & $\begin{array}{c}\text { Performance seems to increase } 6 \\
\text { months post-operation. }\end{array}$ \\
\hline
\end{tabular}


Table 1. Cont.

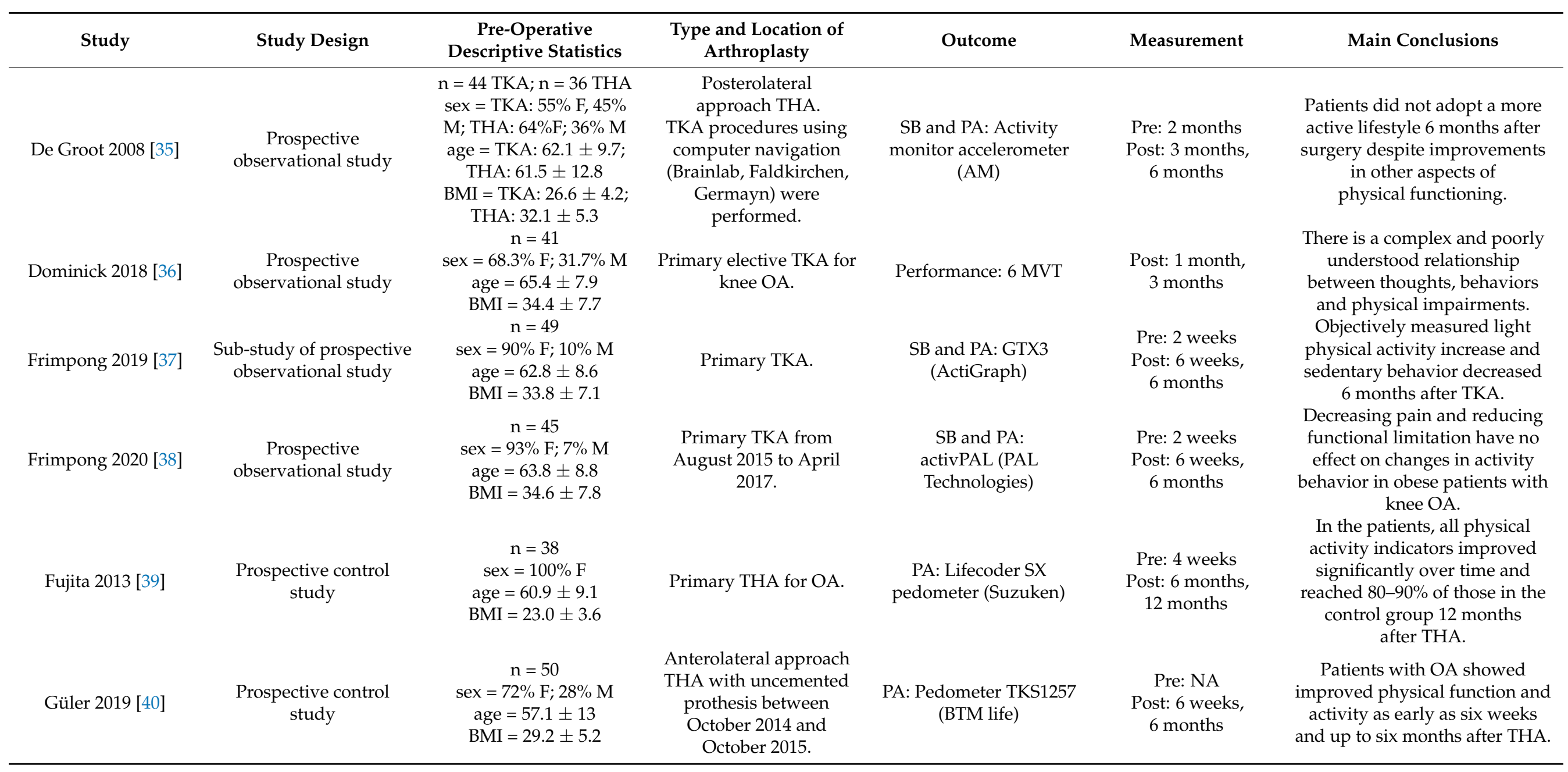


Table 1. Cont.

\begin{tabular}{|c|c|c|c|c|c|c|}
\hline Study & Study Design & $\begin{array}{c}\text { Pre-Operative } \\
\text { Descriptive Statistics }\end{array}$ & $\begin{array}{l}\text { Type and Location of } \\
\text { Arthroplasty }\end{array}$ & Outcome & Measurement & Main Conclusions \\
\hline Hawke 2019 [41] & $\begin{array}{c}\text { Prospective } \\
\text { observational study }\end{array}$ & $\begin{array}{c}\mathrm{n}=54 \\
\text { sex }=57 \% \mathrm{~F} ; 43 \% \mathrm{M} \\
\text { age }=67.8 \pm 8 \\
\mathrm{BMI}=\mathrm{N} / \mathrm{A}\end{array}$ & $\begin{array}{l}\text { Primary total hip or knee } \\
\text { joint replacement. }\end{array}$ & $\begin{array}{l}\text { Performance: } \\
\text { 6MWT, TUG }\end{array}$ & $\begin{array}{c}\text { Post: } 0 \text { weeks, } \\
6 \text { weeks, } 12 \text { weeks }\end{array}$ & $\begin{array}{l}\text { Walking performance increased } \\
\text { after group-based therapy and } \\
\text { continued to improve after } \\
\text { group discharge. }\end{array}$ \\
\hline Heiberg 2013 [42] & $\begin{array}{c}\text { Prospective } \\
\text { observational study }\end{array}$ & $\begin{array}{c}\mathrm{n}=88 \\
\text { sex }=58 \% \mathrm{~F} ; 42 \% \mathrm{M} \\
\text { age }=66(64-68) \\
\mathrm{BMI}=27(26-27)\end{array}$ & $\begin{array}{l}\text { Primary THA from } \\
\text { October } 2008 \text { to } \\
\text { June } 2010 .\end{array}$ & Performance: 6MWT & $\begin{array}{l}\text { Pre: } \\
\text { Post: } 3 \text { months, } \\
12 \text { months }\end{array}$ & $\begin{array}{l}\text { After THA performance } \\
\text { improved slowly through the } \\
\text { first post-operative year. }\end{array}$ \\
\hline Höll 2018 [43] & $\begin{array}{c}\text { Prospective } \\
\text { observational study }\end{array}$ & $\begin{array}{c}\mathrm{n}=46 \\
\text { sex }=54.4 \% \mathrm{~F} ; 45.6 \% \mathrm{M} \\
\text { age }=63.3 \pm 10 \\
\mathrm{BMI}=27.1 \pm 4\end{array}$ & $\begin{array}{l}\text { Minimal invasive, direct } \\
\text { anterior approach THA. }\end{array}$ & $\begin{array}{l}\text { PA and SB: Step-Watch } \\
\text { 3TM Activity Monitor } \\
\text { (Orthocare Innovations) }\end{array}$ & $\begin{array}{l}\text { Pre: } 1 \text { week } \\
\text { Post: } 6 \text { weeks, } \\
3 \text { months }\end{array}$ & $\begin{array}{c}\text { Objectively measured PA takes } \\
\text { longer than } 6 \text { weeks for } \\
\text { significant improvements. }\end{array}$ \\
\hline Jeldi 2017 [44] & $\begin{array}{c}\text { Prospective } \\
\text { observational study }\end{array}$ & $\begin{array}{c}\mathrm{n}=30 \\
\text { sex }=70 \% \mathrm{~F} ; 30 \% \mathrm{M} \\
\text { age }=67(50-82) \\
\mathrm{BMI}=31(19-43)\end{array}$ & $\begin{array}{l}\text { Uncemented an } \\
\text { cemented posterior } \\
\text { approach THA. }\end{array}$ & $\begin{array}{l}\text { PA and SB: } \\
\text { activPAL3 (PAL } \\
\text { Technologies) }\end{array}$ & $\begin{array}{l}\text { Pre: within } 2 \text { weeks } \\
\text { Post: } 3 \text { months, } \\
12 \text { months }\end{array}$ & $\begin{array}{l}\text { No change in the volume of PA } \\
12 \text { months post-operation. }\end{array}$ \\
\hline Kahn 2015 [45] & Cross section study & $\begin{array}{c}\mathrm{n}=63 ; \mathrm{n}=60 \\
\text { sex }=49.2 \% \mathrm{~F} ; 50.8 \% \\
50 \% \mathrm{~F} ; 50 \% \mathrm{M} \\
\text { age }=68.4 \pm 8.2 \\
67.3 \pm 8.7 \\
\mathrm{BMI}=29.2 \pm 4.8 \\
31.1 \pm 5.3\end{array}$ & Primary TKA. & $\begin{array}{l}\text { PA and SB: } \\
\text { ActiGraph GT1M } \\
\text { (ActiGraph) }\end{array}$ & $\begin{array}{c}\text { Pre: } \\
552.6 \pm 358.9 \text { days } \\
\text { Post: } \\
624.8 \pm 420.6 \text { days }\end{array}$ & $\begin{array}{l}\text { No significant difference in } \\
\text { physical activity levels between } \\
\text { the OA and TKA group. }\end{array}$ \\
\hline Ko 2013 [46] & $\begin{array}{l}\text { Randomized control } \\
\text { study }\end{array}$ & $\begin{array}{c}\mathrm{n}=32 \\
\text { sex }=56 \% \mathrm{~F} ; 44 \% \mathrm{M} \\
\text { age }=66.7(64.3-69.2) \\
\mathrm{BMI}=30.8(27.6-34.9)\end{array}$ & Primary TKA. & $\begin{array}{c}\text { Performance: } 6 \mathrm{MWT} \text {, } \\
\text { TUG }\end{array}$ & Post: 12 to 18 months & $\begin{array}{l}\text { Controls performed significantly } \\
\text { better in both the TUG and } \\
6 \mathrm{MWT} \text {. }\end{array}$ \\
\hline Kuhn 2013 [47] & $\begin{array}{c}\text { Prospective } \\
\text { observational study }\end{array}$ & $\begin{array}{c}\mathrm{n}=37 \\
\text { sex }=68 \% \mathrm{~F} ; 32 \% \mathrm{M} \\
\text { age }=42.1 \pm 7.2 \\
\mathrm{BMI}=29 \pm 5.6\end{array}$ & Primary THA. & $\begin{array}{l}\text { PA and SB: } \\
\text { StepWatch Activity } \\
\text { Monitor } 3.0 \\
\text { (Cyma Corp.) }\end{array}$ & $\begin{array}{c}\text { Pre: NA } \\
\text { Post: } 1.3 \pm 0.2 \text { years }\end{array}$ & $\begin{array}{l}\text { Significant improvement in } \\
\text { physical activity level and } \\
\text { intensity was observed } \\
\text { in patients. }\end{array}$ \\
\hline
\end{tabular}


Table 1. Cont.

\begin{tabular}{|c|c|c|c|c|c|c|}
\hline Study & Study Design & $\begin{array}{c}\text { Pre-Operative } \\
\text { Descriptive Statistics }\end{array}$ & $\begin{array}{l}\text { Type and Location of } \\
\text { Arthroplasty }\end{array}$ & Outcome & Measurement & Main Conclusions \\
\hline Lin 2013 [48] & $\begin{array}{c}\text { Prospective } \\
\text { observational study }\end{array}$ & $\begin{array}{c}\mathrm{n}=12 \\
\text { sex }=100 \% \mathrm{~F} \\
\text { age }=58.2 \pm 3.7 \\
\mathrm{BMI}=23.4 \pm 4.1\end{array}$ & $\begin{array}{l}\text { THA using Secur-Fit } \\
\text { Plus Max stem (Stryker), } \\
\text { Trident Acetabular Shell } \\
\text { (Stryker) and Trident } \\
\text { Polyethylene Bearing } \\
\text { (Stryker) implants. }\end{array}$ & $\begin{array}{l}\text { PA and SB: RT3 } \\
\text { accelerometer } \\
\text { (StayHealthy) }\end{array}$ & $\begin{array}{l}\text { Pre: } 1 \text { month } \\
\text { Post: } 6 \text { months }\end{array}$ & $\begin{array}{l}\text { Patients did not develop a more } \\
\text { active lifestyle, but they } \\
\text { increased the amount of } \\
\text { moderate and vigorous } \\
\text { activities after surgery. }\end{array}$ \\
\hline Lützner 2014 [49] & $\begin{array}{c}\text { Prospective } \\
\text { observational study }\end{array}$ & $\begin{array}{c}\mathrm{n}=97 \\
\text { sex }=46.4 \% \mathrm{~F} ; 53.6 \% \mathrm{M} \\
\text { age }=68.9(67.2-70.6) \\
\mathrm{BMI}=31.3(30.3-32.3)\end{array}$ & $\begin{array}{l}\text { Unconstrained TKA } \\
\text { between March } 2009 \text { and } \\
\text { September } 2011 .\end{array}$ & $\begin{array}{l}\text { PA and SB: } \\
\text { activPAL (PAL } \\
\text { Technologies) }\end{array}$ & $\begin{array}{c}\text { Pre: } 1 \text { week } \\
\text { Post: } 12 \text { months }\end{array}$ & $\begin{array}{l}\text { Moderate improvement in the } \\
\text { total number of steps, but no } \\
\text { change in daily walking time. }\end{array}$ \\
\hline Lützner 2016 [50] & $\begin{array}{c}\text { Prospective } \\
\text { observational study }\end{array}$ & $\begin{array}{c}\mathrm{n}=221 \\
\text { sex }=56.6 \% \mathrm{~F} ; 43.4 \% \mathrm{M} \\
\text { age }=68.1 \pm 9.5 \\
\mathrm{BMI}=31.3 \pm 4.9\end{array}$ & $\begin{array}{l}\text { Unconstrained } \\
\text { bicondylar TKA. }\end{array}$ & $\begin{array}{l}\text { PA and SB: } \\
\text { activPAL (PAL } \\
\text { Technologies) }\end{array}$ & $\begin{array}{l}\text { Pre: } 1 \text { weekPost: } \\
12 \text { months }\end{array}$ & $\begin{array}{l}\text { MVPA and the number of steps } \\
\text { were significantly increased } \\
12 \text { months follow up. }\end{array}$ \\
\hline $\begin{array}{l}\text { Matsunaga-Myoji } \\
2019[51]\end{array}$ & $\begin{array}{l}\text { Prospective control } \\
\text { study }\end{array}$ & $\begin{array}{c}\mathrm{n}=66 \\
\text { sex }=83 \% \mathrm{~F} ; 17 \% \mathrm{M} \\
\text { age }=73.3 \pm \mathrm{N} / \mathrm{A} \\
\mathrm{BMI}=\mathrm{N} / \mathrm{A}\end{array}$ & $\begin{array}{l}\text { Primary TKA between } \\
\text { March } 2010 \text { and } \\
\text { November } 2013 .\end{array}$ & $\begin{array}{l}\text { PA and SB: Lifecorder } \\
\text { EX (Suzuken Co.) }\end{array}$ & $\begin{array}{l}\text { Pre: } 1 \text { month } \\
\text { Post: } 6 \text { months }\end{array}$ & $\begin{array}{l}\text { TKA in older patients led to an } \\
\text { increase in the amount of PA. }\end{array}$ \\
\hline $\begin{array}{l}\text { Matsunaga-Myoji } \\
\text { 2020a [52] }\end{array}$ & $\begin{array}{c}\text { Prospective } \\
\text { observational study }\end{array}$ & $\begin{array}{c}\mathrm{n}=153 \\
\text { sex }=84.1 \% \mathrm{~F} ; 15.9 \% \mathrm{M} \\
\text { age }=61.8 \pm 7.9 \\
\mathrm{BMI}=23 \pm 3.5\end{array}$ & $\begin{array}{l}\text { Primary TKA between } \\
\text { March } 2010 \text { and } \\
\text { November } 2013 .\end{array}$ & $\begin{array}{l}\text { PA and SB: Lifecorder } \\
\text { EX (Suzuken Co.) }\end{array}$ & $\begin{array}{l}\text { Pre: } 1 \text { month } \\
\text { Post: } 6 \text { months, } \\
24 \text { months }\end{array}$ & $\begin{array}{l}\text { MVPA and the number of steps } \\
\text { was significantly increased } \\
1 \text { year after the operation. }\end{array}$ \\
\hline $\begin{array}{l}\text { Matsunaga-Myoji } \\
\text { 2020b [53] }\end{array}$ & $\begin{array}{c}\text { Prospective } \\
\text { observational study }\end{array}$ & $\begin{array}{c}\mathrm{n}=58 \\
\text { sex }=84.5 \% \mathrm{~F} ; 15.5 \% \mathrm{M} \\
\text { age }=72.6 \pm 6 \\
\mathrm{BMI}=26.1 \pm 4.4\end{array}$ & $\begin{array}{l}\text { Primary THA between } \\
\text { October } 2010 \text { and } \\
\text { November } 2011 .\end{array}$ & $\begin{array}{l}\text { PA and SB: Lifecorder } \\
\text { EX (Suzuken Co.) }\end{array}$ & $\begin{array}{l}\text { Pre: } 1 \text { month } \\
\text { Post: } 6 \text { months, } \\
46 \text { months and } \\
60 \text { months }\end{array}$ & $\begin{array}{l}\text { PA after } 6 \text { months exhibited } \\
\text { pre-operation levels. Only } \\
\text { MVPA increased between } 2 \text { and } \\
6 \text { months. Patients can expect } \\
\text { PA to continue to improve up to } \\
2 \text { years after TKA. }\end{array}$ \\
\hline $\begin{array}{l}\text { Moellenbeck } \\
\text { 2020a [54] }\end{array}$ & $\begin{array}{c}\text { Prospective } \\
\text { observational study }\end{array}$ & $\begin{array}{c}\mathrm{n}=16 \\
\text { sex }=43.8 \% \mathrm{~F} ; 56.2 \% \mathrm{M} \\
\text { age }=68.9 \pm 6.8 \\
\mathrm{BMI}=26.4 \pm 4.3\end{array}$ & Elective THA. & $\begin{array}{c}\text { PA and SB: } \\
\text { ActiGraph wGTX3-BT } \\
\text { Firmware 1.9.2 } \\
\text { (ActiGraph LLC) }\end{array}$ & $\begin{array}{c}\text { Pre: NA } \\
\text { Post: } \\
8.9 \pm 2.3 \text { months }\end{array}$ & $\begin{array}{l}\text { Sedentary behavior did not } \\
\text { show any post-operative } \\
\text { change, even though short } \\
\text { interruptions of sedentary } \\
\text { activity were taken into account }\end{array}$ \\
\hline
\end{tabular}


Table 1. Cont.

\begin{tabular}{|c|c|c|c|c|c|c|}
\hline Study & Study Design & $\begin{array}{c}\text { Pre-Operative } \\
\text { Descriptive Statistics }\end{array}$ & $\begin{array}{l}\text { Type and Location of } \\
\text { Arthroplasty }\end{array}$ & Outcome & Measurement & Main Conclusions \\
\hline $\begin{array}{l}\text { Moellenbeck } \\
\text { 2020b [55] }\end{array}$ & $\begin{array}{c}\text { Prospective } \\
\text { observational study }\end{array}$ & $\begin{array}{c}\mathrm{n}=24 \\
\text { sex }=54 \% \mathrm{~F} ; 46 \% \mathrm{M} \\
\text { age }=69.4 \pm 7.2 \\
\mathrm{BMI}=26.6 \pm 3.8\end{array}$ & $\begin{array}{c}\text { Conventional TKA or } \\
\text { THA. }\end{array}$ & $\begin{array}{c}\text { PA and SB: } \\
\text { ActiGraph wGTX3-BT } \\
\text { Firmware 1.9.2 } \\
\text { (ActiGraph LLC) }\end{array}$ & $\begin{array}{c}\text { Pre: } 2-3 \text { weeks } \\
\text { Post: } \\
12.4 \pm 1.2 \text { months }\end{array}$ & $\begin{array}{c}\text { The habitual activity of patients } \\
\text { stayed the same one year after } \\
\text { the operation. }\end{array}$ \\
\hline Oka 2020 [56] & $\begin{array}{c}\text { Prospective } \\
\text { observational study }\end{array}$ & $\begin{array}{c}\mathrm{n}=82 \\
\text { sex }=82 \% \mathrm{~F} ; 18 \% \mathrm{M} \\
\text { age }=72.1 \pm 5.9 \\
\mathrm{BMI}=26.1 \pm 3.7\end{array}$ & $\begin{array}{l}\text { Primary TKA with } \\
\text { medial parapatellar } \\
\text { approach between June } \\
2016 \text { and June } 2019 \text {. }\end{array}$ & $\begin{array}{c}\text { PA and SB: Active Style } \\
\text { Pro HJA-350IT (Omron } \\
\text { Healthcare) }\end{array}$ & $\begin{array}{l}\text { Pre: } 1 \text { month } \\
\text { Post: } 3 \text { months and } \\
6 \text { months }\end{array}$ & $\begin{array}{l}\text { Physical activity level was not } \\
\text { increased after operation. }\end{array}$ \\
\hline Rezzadeh 2019 [57] & $\begin{array}{c}\text { Prospective } \\
\text { observational study }\end{array}$ & $\begin{array}{c}\mathrm{n}=18 \\
\text { sex }=50 \% \mathrm{~F} ; 50 \% \mathrm{M} \\
\text { age }=66.3 \pm 9.4 \\
\mathrm{BMI}=28.7 \pm 4.5 \\
\mathrm{n}=\mathrm{A}: 29 ; \mathrm{B}: 4 ; \mathrm{C}: 18\end{array}$ & Unilateral TKA. & $\begin{array}{l}\text { PA and SB: } \\
\text { Accelerometer (not } \\
\text { described) }\end{array}$ & $\begin{array}{c}\text { Pre: } \\
185.8 \pm 141.6 \text { days } \\
\text { Post: } \\
544.2 \pm 141.6 \text { days }\end{array}$ & $\begin{array}{l}\text { No significant difference } \\
\text { between post-operative and } \\
\text { pre-operative patients. }\end{array}$ \\
\hline Thewlis 2019 [58] & $\begin{array}{c}\text { Prospective } \\
\text { observational study }\end{array}$ & $\begin{array}{c}\text { sex }=\text { NA } \\
\text { age =A: 63 (24-87); B: } 69 \\
(64-77) ; \text { C: } 65 \text { (41-83) } \\
\text { BMI = A: 30.8 (21.4-40.7); } \\
\text { B: 23.6 (22.8-24.4); C: } \\
28.9(18.6-40.0) ; \mathrm{n}=58\end{array}$ & $\begin{array}{l}\text { Primary THA between } \\
\text { August } 2016 \text { and } \\
\text { February } 2018 .\end{array}$ & $\begin{array}{l}\text { PA and SB: Wrist worn } \\
\text { accelerometer } \\
\text { (GeneActiv) }\end{array}$ & $\begin{array}{l}\text { Pre: within } 4 \text { weeks } \\
\text { Post: } 2 \text { weeks, } \\
6 \text { weeks, } 12 \text { weeks, } \\
26 \text { weeks }\end{array}$ & $\begin{array}{l}\text { Physical activity did not } \\
\text { significantly increase } \\
\text { after operation. }\end{array}$ \\
\hline Tobinaga 2019 [59] & $\begin{array}{c}\text { Prospective } \\
\text { observational study }\end{array}$ & $\begin{array}{c}\text { sex }=84.5 \% \mathrm{~F} ; 15.5 \% \mathrm{M} \\
\text { age }=74.6 \pm 6.5 \\
\mathrm{BMI}=26.3 \pm 4.2\end{array}$ & Unilateral TKA. & Performance: TUG & $\begin{array}{c}\text { Pre: NA } \\
\text { Post: } 3 \text { months }\end{array}$ & $\begin{array}{l}\text { Performance was significantly } \\
\text { increased after } 3 \text { months. }\end{array}$ \\
\hline Twiggs 2018 [60] & $\begin{array}{c}\text { Prospective } \\
\text { observational study }\end{array}$ & $\begin{array}{c}\mathrm{n}=91 \\
\text { sex }=50.5 \% \mathrm{~F} ; 49.5 \% \mathrm{M} \\
\text { age }=67.5 \pm 13.1 \\
\mathrm{BMI}=30.1 \pm 6.3\end{array}$ & $\begin{array}{l}\text { TKA over } 21 \text { months } \\
\text { period between } \\
\text { December } 2013 \text { and } \\
\text { September } 2015 .\end{array}$ & $\begin{array}{l}\text { PA and SB: tri-axial } \\
\text { accelerometer FitBit Flex }\end{array}$ & $\begin{array}{c}\text { Pre: } 2 \text { weeks } \\
\text { Post: } 1 \text { day, } 6 \text { weeks }\end{array}$ & $\begin{array}{l}\text { The number of steps did not } \\
\text { increase after } 6 \text { weeks } \\
\text { post-operation. }\end{array}$ \\
\hline Vissers 2013 [61] & $\begin{array}{c}\text { Prospective } \\
\text { observational study }\end{array}$ & $\begin{array}{c}\mathrm{n}=44 \\
\text { sex }=59.1 \% \mathrm{~F} ; 40.9 \% \mathrm{M} \\
\text { age }=63.8 \pm 9.4 \\
\mathrm{BMI}=29.7 \pm 5\end{array}$ & $\begin{array}{c}\text { Posterolateral } \\
\text { approach THA. } \\
\text { TKA procedures using } \\
\text { computer navigation } \\
\text { (Brainlab, Faldkirchen, } \\
\text { Germayn) were } \\
\text { performed. }\end{array}$ & $\begin{array}{l}\text { PA and SB: Rotterdam } \\
\text { Activity Monitor-AM } \\
\text { (Vitaport Technology } \\
\text { Temec Instruments) }\end{array}$ & $\begin{array}{l}\text { Pre: } 2 \text { months } \\
\text { Post: } 6 \text { months, } \\
48 \text { months }\end{array}$ & $\begin{array}{l}\text { Performance was significantly } \\
\text { increased after the operation. }\end{array}$ \\
\hline
\end{tabular}

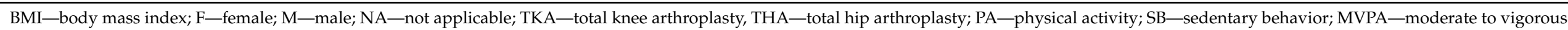
physical activity; TUG—-timed up and go test; $6 \mathrm{MWT}-6$-min walking test. 


\subsection{Comparison of PA between Patients and Healthy Individuals Pre- and Post-Operation}

In the first set of analyses (Table 2), we compared patients with matched control groups pre-operation. There was a very large difference in the number of steps (Figure 2; $\mathrm{SMD}=-1.02 ; p<0.001$ ), with the patients performing, on average, 2892.2 less steps per day compared to the control group. In the second set of analyses, we compared patients with matched control groups 12 or more months post-operation. The amount of moderate to vigorous physical activity (MVPA) $(\mathrm{SMD}=-0.97)$, as well as the number of steps (SMD $=-0.75)$, tended to be lower in patients. However, despite large effect sizes, the differences were not statistically significant $(p=0.180-0.190)$, likely due to the large heterogeneity between studies $\left(\mathrm{I}^{2}=94-97 \%\right)$.

Table 2. Comparison of patients and matched controls in PA, pre-operation and 12 months post-operation.

\begin{tabular}{|c|c|c|c|c|c|c|c|}
\hline $\begin{array}{c}\text { Time after the } \\
\text { Operation }\end{array}$ & Variable & $\operatorname{SMD}(95 \% \mathrm{CI}) *$ & $\begin{array}{c}\text { Studies } \\
\text { (Participants) }\end{array}$ & Effect Size & $p$ & $\mathrm{I}^{2}$ & $\begin{array}{l}\text { Raw Difference } \\
\text { (If Applicable) }\end{array}$ \\
\hline Pre-operation & Number of steps & -1.02 & $4(344)$ & Very large & $<0.001$ & $62 \%$ & -2892.2 steps/day \\
\hline $\begin{array}{l}12 \text { months } \\
\text { post-operation }\end{array}$ & $\begin{array}{c}\text { MVPA } \\
\text { Number of steps }\end{array}$ & $\begin{array}{l}-0.97(-2.39,0.46) \\
-0.75(-1.89,0.38)\end{array}$ & $\begin{array}{l}3(641) \\
3(372)\end{array}$ & $\begin{array}{l}\text { Very large } \\
\text { Large }\end{array}$ & $\begin{array}{l}0.180 \\
0.190\end{array}$ & $\begin{array}{l}97 \% \\
94 \%\end{array}$ & -2671.2 steps/day \\
\hline
\end{tabular}

* Negative SMD—lower value in patients; SMD—standardized mean difference; I ${ }^{2}$-I-squared statistics for study heterogeneity; MVPA— moderate to vigorous physical activity.

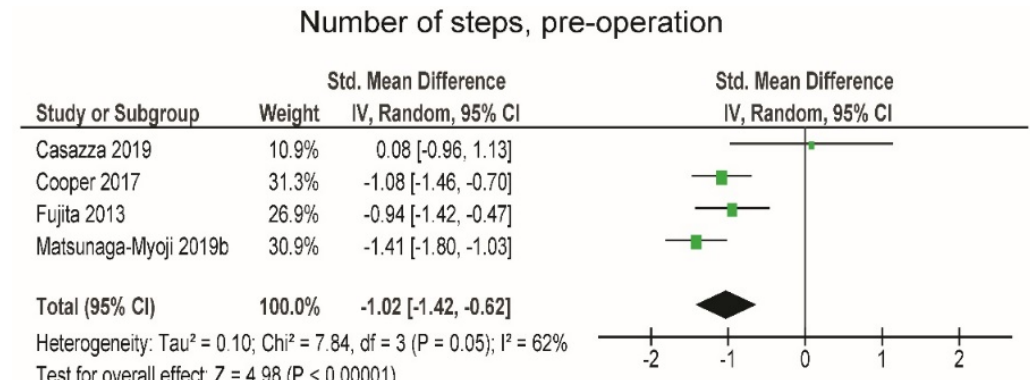

$\begin{array}{lcccc} & \text { Number of steps, post-operation } & \text { Std. Mean Difference } \\ \text { Study or Subgroup } & \text { Weight } & \text { IV, Random, } 95 \% \mathrm{Cl}\end{array}$

Figure 2. Number of steps and moderate to vigorous physical activity in patients and controls, pre-operation and 12 months post-operation. CI—confidence interval; $\mathrm{I}^{2}$ - heterogeneity statistics.

\subsection{Comparison of PA and SB in Patients Pre-and Post-Operation}

Between 3 and 6 months post-operation, MVPA remained at the pre-operative level $(\mathrm{SMD}=0.12, p=0.530)$, increased between 6 and 9 months $(\mathrm{SMD}=0.33, p<0.001)$ and remained greater more than 12 months post-operation (SMD $=0.70, p<0.001$ ) (Figure 3). 


\section{Moderate to vigoruous PA - 3-6 months post operatively}

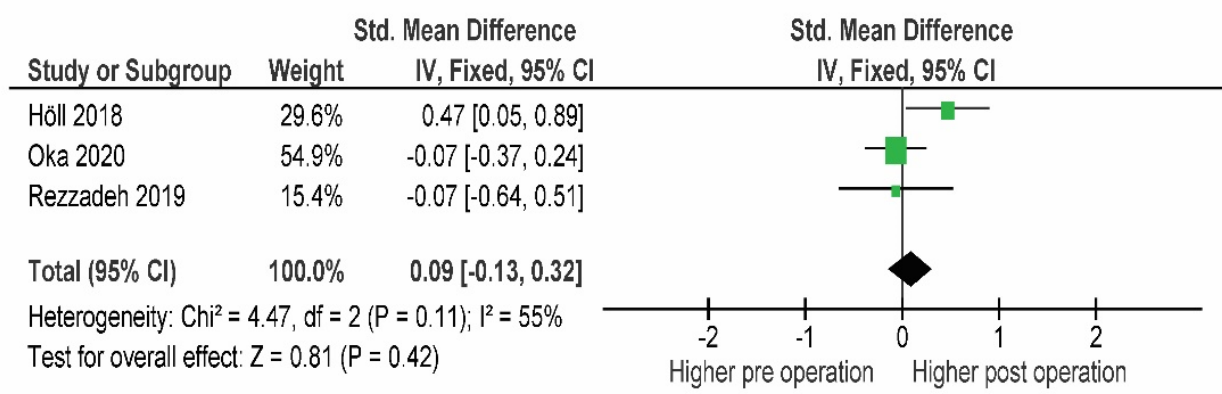

\section{Moderate to vigoruous PA - 6-9 months post operatively}

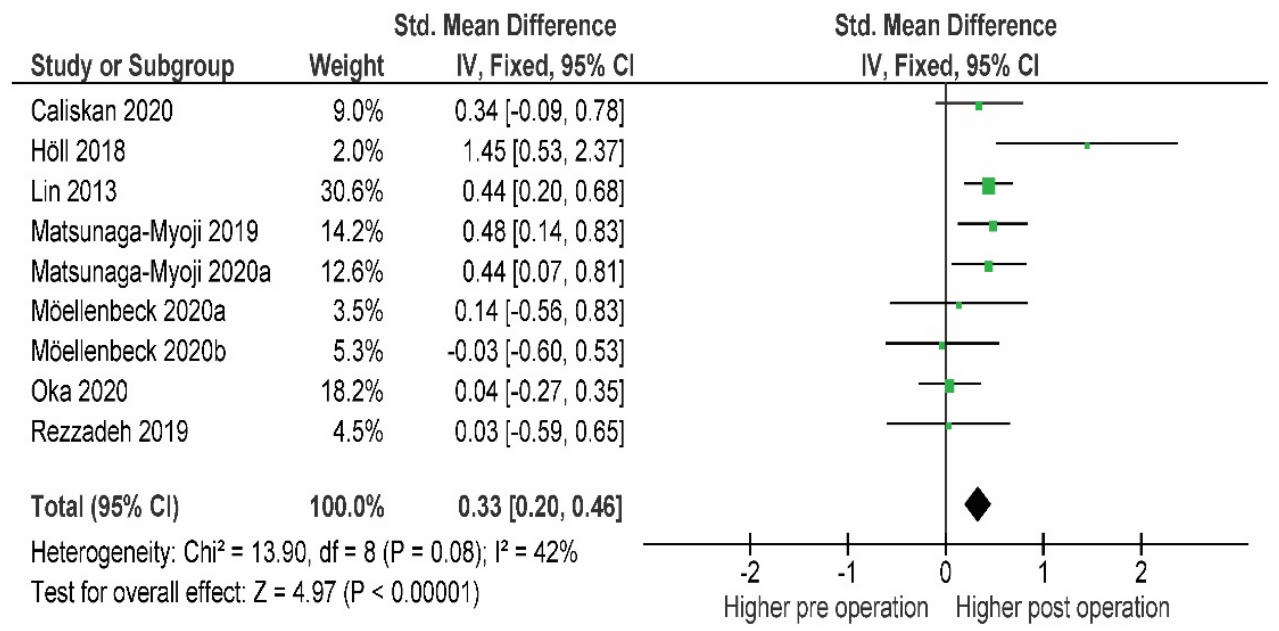

\section{Moderate to vigoruous PA - 12 months post operatively}

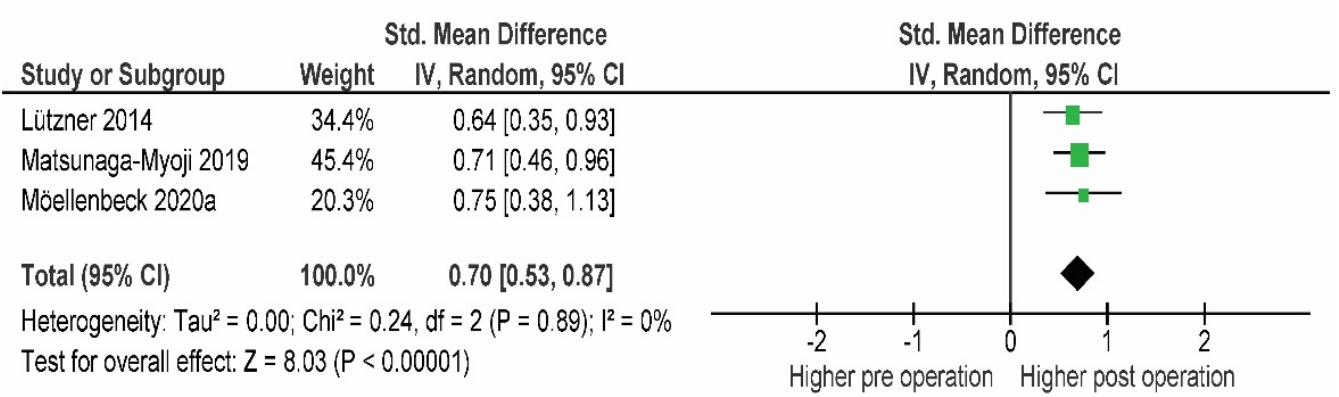

Figure 3. Changes in moderate to vigorous physical activity over the time post-operation. CIconfidence interval; $\mathrm{I}^{2}$ - heterogeneity statistics.

Light-intensity physical activity also tended to increase (SMD $=0.14 ; p=0.160)$, while SB was similar pre-operation and between 6 and 9 months post-operation $(\mathrm{SMD}=-0.00$, $p=0.790)$.

The number of steps $(-196.7$; SMD $=-0.06)$ tended to be lower within 3 months post-operation, but the differences were not statistically significant $(p=0.670)$. Three to six months post-operation, patients slightly but not significantly $(p=0.390)$ increased the number of steps $(+373.8, \mathrm{SMD}=0.15)$. At 6 months, patients significantly exceeded the pre-operative level $(+1064.1, \mathrm{SMD}=0.45, p<0.001)$, which was exceeded 12 months postoperation $(+1425.3, \mathrm{SMD}=0.52, p<0.001)$ (Figure 4$)$. Results regarding sedentary behavior were inconsistent $\left(\mathrm{I}^{2}=53 \%\right)$ and amounted to exactly zero overall effect (SMD $\left.=0.00\right)$ (Figure 5). 


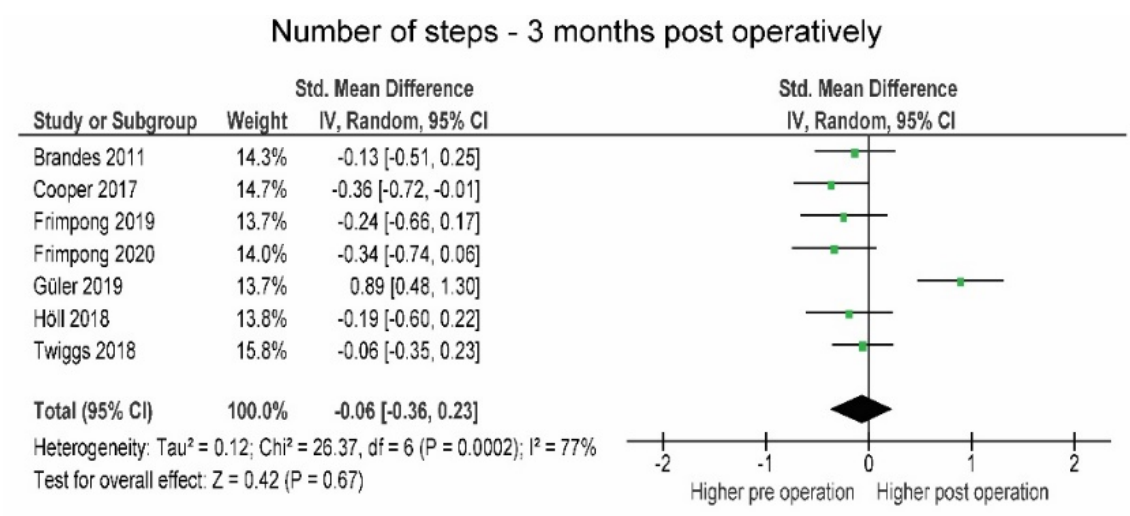

Number of steps - 3-6 months post operatively

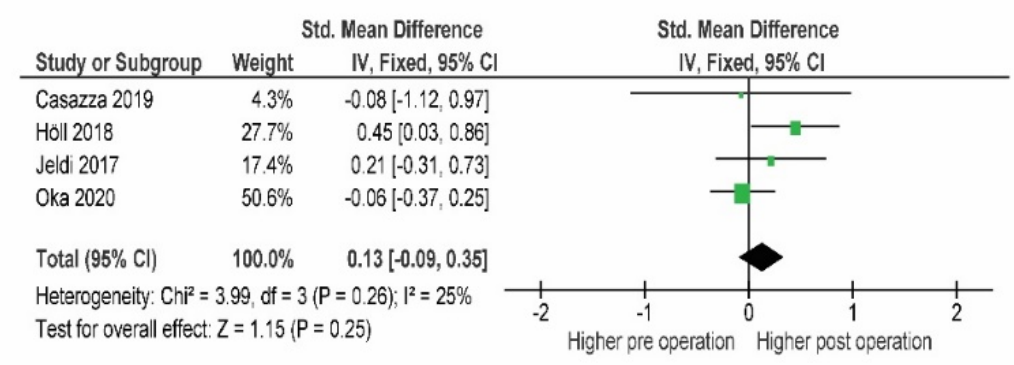

Number of steps - 6-9 months post operatively

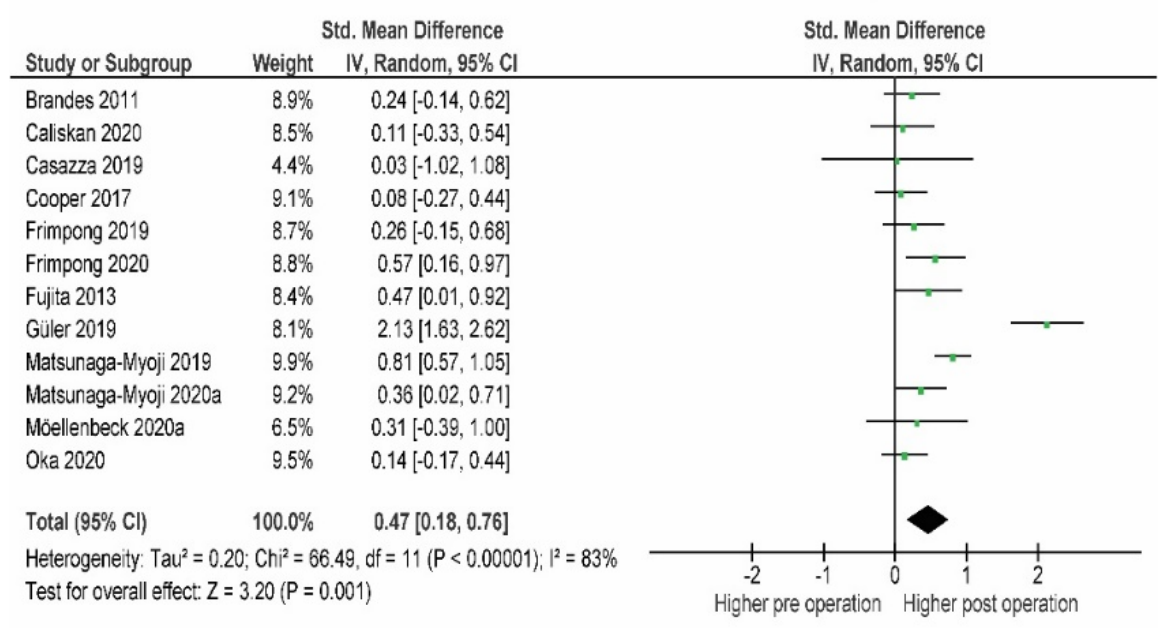

Number of steps - 12 months post operatively

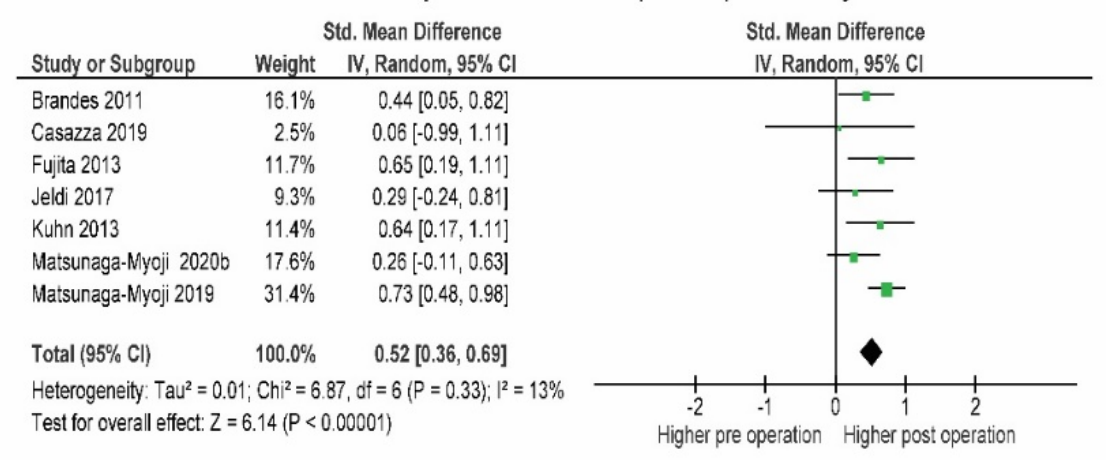

Figure 4. Changes in number of steps over the time post-operation. CI-confidence interval; $\mathrm{I}^{2}-$ heterogeneity statistics. 


\section{Sedentary behaviour}

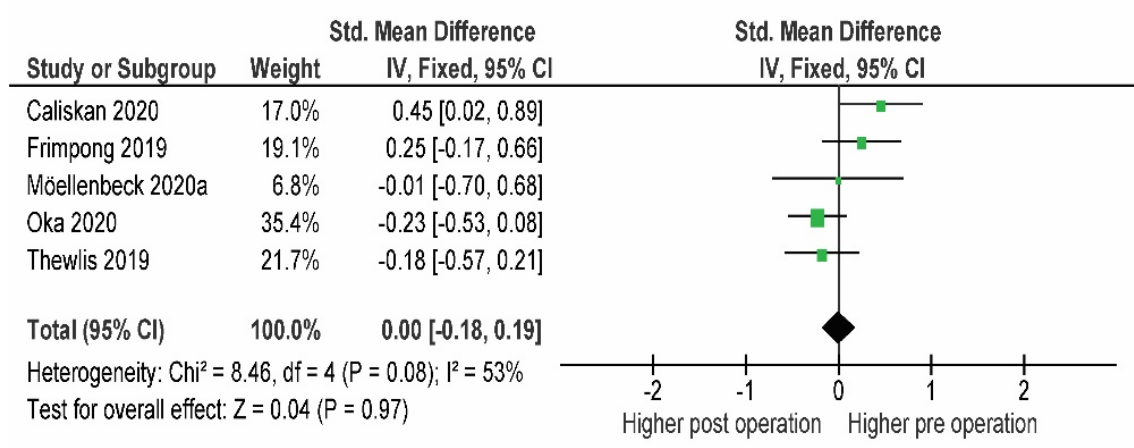

Figure 5. Changes in sedentary behavior 6 to 9 months post-operation. CI-confidence interval; $\mathrm{I}^{2}$ - heterogeneity statistics.

\subsection{Comparison of Functional Performance in Patients Pre-and Post-Operation}

In the first 3 months post-operation, the patients tended to improve their 6MWT $(+59.9 \mathrm{~m}$; SMD $=0.64)$ compared to the pre-operative level, but not enough to confirm this statistically ( $p=0.15$ ) (Figure 6). At this time, the results of the TUG test still indicated a slight, statistically non-significant impairment in function $(+1.58 \mathrm{~s}, \mathrm{SMD}=0.27, p=0.770)$.

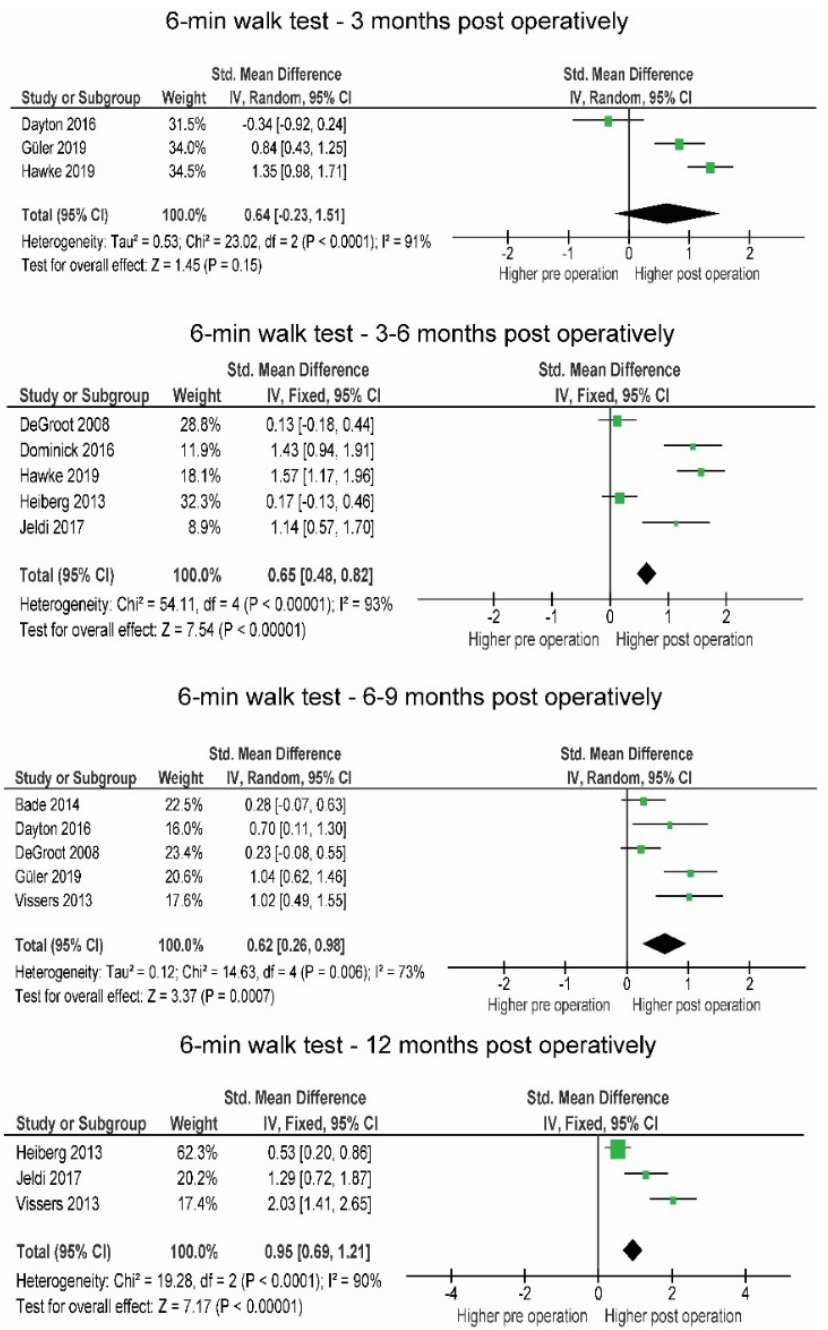

Figure 6. Changes in 6 min walk test over the post-operative time period. CI-confidence interval; $\mathrm{I}^{2}$ - heterogeneity statistics. 
Three months post-operation, the results of the 6MWT (+90.2 $\mathrm{m}$; SMD $=0.87)$ were largely and statistically significantly $(p=0.008)$ improved compared to pre-operation and remained improved between 6 and 9 months $(+71.84 \mathrm{~m}$; SMD $=0.62 ; p<0.001)$. The TUG test was also largely and significantly $(-1.91 \mathrm{~s} ; \mathrm{SMD}=-0.61 ; p<0.001)$ improved between 6 and 9 months compared to pre-operative levels (Figure 7).

\section{Timed up and go test - 3 months post operatively}

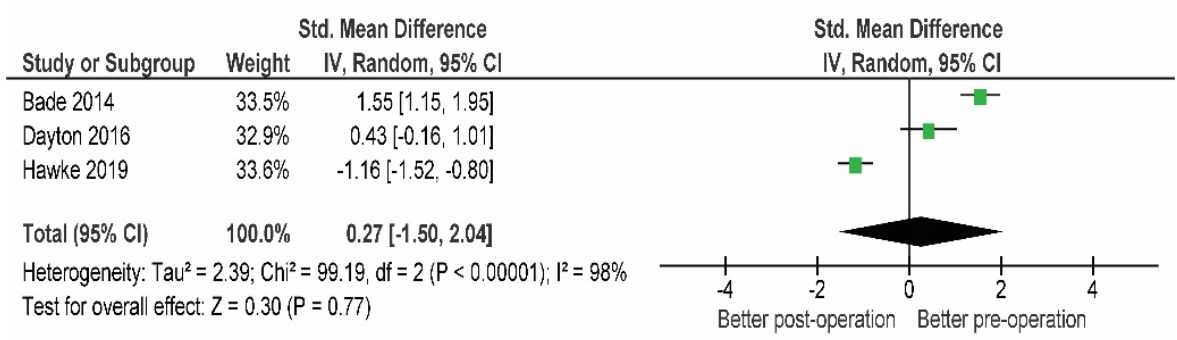

Timed up and go test - 6-9 months post operatively

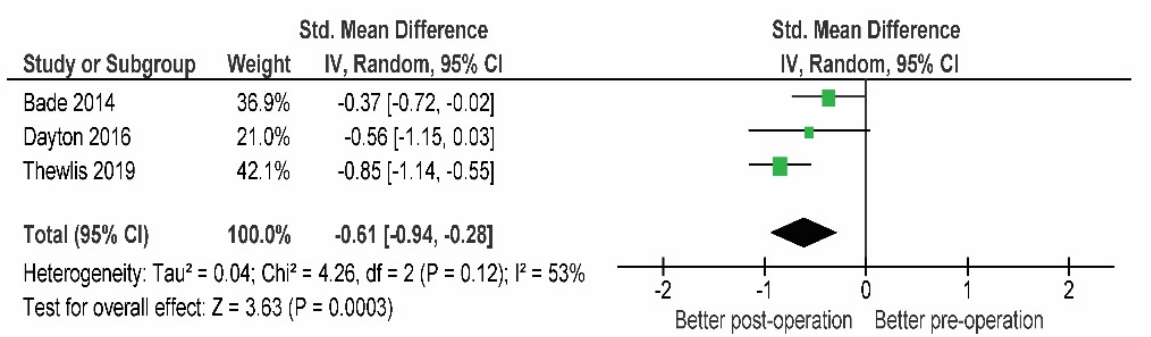

Figure 7. Changes in 6 min walk test over the post-operative time period.

Table 3 summarizes the obtained evidence across the different time intervals.

Table 3. Comparison of the patients in PA, SB and functional performance pre-operation and at $<3$ months, 3-6 months, 6-9 months and $>12$ months post-operation.

\begin{tabular}{|c|c|c|c|c|c|c|}
\hline Variable & $\operatorname{SMD}(95 \% \mathrm{CI}) *$ & $\begin{array}{c}\text { Studies } \\
\text { (Participants) }\end{array}$ & Effect Size & $p$-Value & $\mathbf{I}^{2}$ & $\begin{array}{l}\text { Raw Difference } \\
\text { (If Applicable) }\end{array}$ \\
\hline \multicolumn{7}{|c|}{$<3$ months post-operation } \\
\hline Number of steps & $-0.06(-0.36,0.23)$ & $7(792)$ & Small & 0.670 & $77 \%$ & -196.7 steps/day \\
\hline Timed up and go test & $0.27(-1.50,2.04)$ & $3(314)$ & Small & 0.770 & $98 \%$ & $+1.58 \mathrm{~s}$ \\
\hline 6 Minute Walk Test & $0.64(-0.23,1.51)$ & $4(478)$ & Moderate & 0.150 & $91 \%$ & $+59.9 \mathrm{~m}$ \\
\hline \multicolumn{7}{|c|}{ 3-6 months post-operation } \\
\hline $\begin{array}{l}\text { Moderate to vigorous } \\
\text { physical activity }\end{array}$ & $0.12(-0.25,0.48)$ & $3(301)$ & Small & 0.530 & $55 \%$ & / \\
\hline Number of steps & $0.15(-0.12,0.42)$ & $4(327)$ & Small & 0.390 & $25 \%$ & +373.8 steps/day \\
\hline 6 Minute Walk Test & $0.65(0.48,0.82)$ & $5(605)$ & Large & $<0.01$ & $93 \%$ & $+90.2 \mathrm{~m}$ \\
\hline \multicolumn{7}{|c|}{ 6-9 months post-operation } \\
\hline $\begin{array}{l}\text { Moderate to vigorous } \\
\text { physical activity }\end{array}$ & $0.33(0.20,0.46)$ & $9(923)$ & Moderate & $<0.001$ & $42 \%$ & / \\
\hline $\begin{array}{l}\text { Light-intensity } \\
\text { physical activity }\end{array}$ & $0.14(0.06,0.35)$ & $8(889)$ & Small & 0.160 & $49 \%$ & / \\
\hline Sedentary behavior & $-0.00(-0.18,0.19)$ & $5(473)$ & Small & 0.790 & $53 \%$ & $-3.72 \mathrm{~min} /$ day \\
\hline Number of steps & $0.47(0.18,0.76)$ & $12(1418)$ & Moderate & $<0.001$ & $83 \%$ & +1064.1 steps / day \\
\hline Timed-up and go test & $-0.61(-0.94,-0.28)$ & $3(364)$ & Large & $<0.001$ & $53 \%$ & $-1.91 \mathrm{~s}$ \\
\hline 6 Minute Walk Test & $0.62(0.26,0.98)$ & $5(493)$ & Large & $<0.001$ & $73 \%$ & $+71.84 \mathrm{~m}$ \\
\hline
\end{tabular}


Table 3. Cont.

\begin{tabular}{ccccccc}
\hline Variable & SMD (95\% CI) & $\begin{array}{c}\text { Studies } \\
\text { (Participants) }\end{array}$ & Effect Size & $p$-Value & I $^{2}$ & $\begin{array}{c}\text { Raw Difference } \\
\text { (If Applicable) }\end{array}$ \\
\hline & $>12$ months post-operation & & & \\
\hline $\begin{array}{c}\text { Moderate to vigorous } \\
\text { physical activity }\end{array}$ & $0.70(0.53,0.87)$ & $3(573)$ & Large & $<0.001$ & $0 \%$ & \\
Number of steps & $0.52(0.36,0.69)$ & $7(707)$ & Moderate & $<0.001$ & $13 \%$ & +1425.3 steps $/$ say \\
\hline
\end{tabular}

* Positive SMD—higher values post-operation. SMD—standardized mean difference; $\mathrm{I}^{2}$ —I-squared statistics for study heterogeneity.

\subsection{Sensitivity Analyis}

According to the sensitivity analyses for all outcomes at all time points, no single studies were identified that would change the statistical significance of the pooled effect size.

\section{Discussion}

The primary aim of this systematic review was to determine whether total arthroplasty is associated with changes in objectively measured PA, SB and functional performance at different post-operation follow-up periods to examine the progression of recovery after lower limb arthroplasty.

\subsection{Comparison of PA between Patients and Healthy Subjects Pre-and 12 Months Post-Operation}

Patients were less physically active than healthy peers both pre- and 12 months post-operation (Figure 2). Specifically, a lower number of steps was observed in patients pre-operation. Studies by Fujita et al. [39] and Matsunaga-Myoji et al. [51] suggest that the level of light PA is also lower in OA patients awaiting surgery than in healthy individuals. In addition, Moellenbeck et al. [55] and Matsunaga-Myoji et al. [51] found that healthy subjects spend more time doing MVPA than OA patients. The observed trend could be a logical consequence of OA symptoms preventing weight-bearing PA and increasing SB. It seems that the discrepancy in MVPA and performance between controls and patients is still evident 12 months or more after total joint arthroplasty [46,62]. Although OA pain has been shown to be decrease post-operation, patients do not reach the performance and activity levels of healthy individuals even at 12 months or more post-operation.

\subsection{Comparison in PA, SB and Performance of Patients Pre-and Post-Operation}

Prospective observations of patients are more numerous in the literature than comparisons with healthy controls. In this section, pre-operative PA and SB levels as well as functional performance at different time intervals were compared to post-operative levels.

\subsubsection{Post-Operative Changes in PA}

In the first three months post-operation, the number of steps remained the same as those observed pre-operation. When the study by Güler et al. [40] is excluded from the analysis, significantly more steps are taken pre-operation (Figure 2). Nevertheless, it may not be reliable to draw conclusions about the general level of PA based on the number of steps, as steps are only a partial survey of the frequency of physical activity. Intensity and duration need to be recorded to more accurately assess the level of PA. In the prospective study, Frimpong et al. [37] observed that within the first three months post-operation, patients spent $27 \mathrm{~min}$ per day less engaging in moderate intensity PA than pre-operation and maintained the level of SB. Similarly, Höll et al. [43] observed that about 300 fewer steps per day were taken shortly after surgery at light-intensity PA and also noted a slight increase in MVPA. When making comparisons between studies, we must consider that the measurements were taken at different post-operative times: 2 and 10 weeks. Therefore, those who measured PA earlier likely noted greater impairment due to higher acute inflammatory processes limiting overall activity and function. Intensive physiotherapy during this period must also be taken into account as it may affect the 
patient's overall PA levels. It is very likely that post-operative limitations such as pain and protection of the affected limb affect PA within the first three months post-operation $[63,64]$.

Four studies were included in the analysis of the number of steps and three studies in the analysis of MVPA between 3 and 6 months post-operation (Figures 1 and 2). Very little difference was observed in the number of steps (+333 steps/day), while no difference was observed in MVPA. Only two studies [56,58] observed SB, and in both, patients already reached but did not exceed pre-operative levels. Light-intensity physical activity remained the same, as noted in the studies by Thewlis et al. [58] and Oka et al. [56] (from 329 pre- to $316 \mathrm{~min} /$ day post- and from 239 pre- to $232 \mathrm{~min}$ / day post-, respectively).

At 6 to 9 months post-operation, MVPA and the number of steps were above preoperative levels. These observations are supported by previous findings by Mills et al. [17], who only found a minimal increase in MVPA 6 months post-operation. The slight discrepancy in MVPA between studies could be due to differences in the interpretation of variables describing PA and differences in methodology and the number of studies included in the analyses. The main determinants in the study by Mills et al. [17] were time spent in locomotion and time spent active, while we used MVPA and SB as PA determinants.

The results of the prospective studies with a follow-up period of 12 months or longer showed high homogeneity and a trend toward increased number of steps (Figure 4). Three studies in which MVPA was observed provided a large effect size, indicating a long-term post-operative increase in PA. The results of our study showed that patients fully regained and exceeded their pre-operative PA level after 6 and 12 months. Similar results were previously reported in two systematic reviews by Arnolad et al. [65] and Mills et al. [17], where improvements in PA over time, especially after 12 or more months, were evident.

\subsubsection{Post-Operative Changes in SB}

Three studies compared SB less than 3 months $[37,58]$ and from 3 to 6 months $[56,58]$ post-operation. The time trajectory of SB is clearly seen from the study by Thewlis et al. [58], in which SB increases from pre-operation to 2 weeks post-operation for $120 \mathrm{~min} /$ day and decreases back to pre-operative levels after 12 weeks (620 $\mathrm{min} /$ day to $624 \mathrm{~min} /$ day). A similar trend was observed by Oka et al. [56], while Frimpong et al. [37] reported that SB was already recovered after 6 weeks post-operation.

Most of the SB data were provided from 6 to 9 months post-operation. On average, the values of SB remained constant and did not significantly decrease 6 months post-operation, similar to the study by Frimpong et al. [38] in which only a small decrease in sitting time was observed at 6 months ( $5 \%)$. In general, SB is not significantly reduced in a time period from 6 to 9 months, but in the study by Möellenbeck et al. [54], sedentary activities longer than $60 \mathrm{~min}$ were significantly reduced in older adult patients. As long-term objectively measured SB data are lacking and studies report divergent SB variables, future research should primarily focus on methodological quality and long-term outcomes.

\subsubsection{Post-Operative Changes in Functional Performance}

In the first three months post-operation, 6MWT and TUG increased slightly but not significantly. It is logical that patients regain pre-operative functional performance levels more related to general locomotion ability, such as walking at a steady pace, sooner after arthroplasty than more complex tasks such as explosive strength and agility. The subsequent restoration of TUG could be due to the fact that surgery-related swelling and pain do not enable one to perform at peak functional capacity since strength deficits of lower limbs might be present [66].

At 3 to 6 months, the 6MWT was significantly higher than the pre-operation, suggesting that the surgically induced pain might be reduced and functional capacity restored by arthroplasty [66]. It has been reported that the subjective perception of functional ability at this time period was lower than pre-operation [67], so there may be some discrepancies between actual functional ability and the subjective perception of functional ability due to post-operative anxiety and uncertainty of the patients. Our results showed that six 
months post-operation, patients exceed their pre-operation functional ability. Restored functional capacity allows patients to participate in sport, as Witjes et al. [68] reported that the majority of patients after lower limb arthroplasty who were previously physically active returned to high-impact sports after 26 weeks.

\subsubsection{Coherent Interpretation of PA, SB and Functional Performance Changes}

Overall, objectively measured PA appears to increase over time, but patients are unable to reach pre-operative levels of PA until about 6 months post-operation, when PA values match and then exceed pre-operative levels. Functional performance, on the other hand, tends to increase earlier ( 3 months post-operation) and continues to develop up to 12 months post-operation. Although functional performance is restored at 3 months, MVPA remains at pre-operative levels. Moreover, SB remains unchanged in the period between 6 and 9 months. Since the main goals of rehabilitation after joint arthroplasty are to maximize functional performance, optimize lifestyle and promote patient independence to improve overall health [69], increasing PA and reducing SB should be incorporated and encouraged as soon as possible. The results of this study show that even if functional performance is increased, patients remain as sedentary as they were before arthroplasty. Only PA recovered, albeit with a delay, and significantly exceeded the pre-operative level at 12 months post-operation. Although arthroplasty significantly restored patients' function, patients remained less physically active than their healthy peers 12 months post-operation.

According to Bull et al. [11], adults and older adults should spend at least 150-300 min per week in MVPA to achieve substantial health benefits of physical activity. Only in the studies by Moellenback et al. [55], Oka et al. [56] and Hylkema et al. [70] did patients achieve these recommendations 6 and 12 months post-operation. On average, functional performance and PA increased after the arthroplasty, but not enough to reach the general health guidelines. More importantly, SB remained unchanged. Therefore, novel rehabilitation protocols could solve the problem of achieving sufficient PA and reducing SB in the long term. Peter and colleagues [71] already proposed to expand the current recommendations for the rehabilitation process after lower limb arthroplasty. A behavioral approach could be applied in line with recent guidelines, which aim to limit SB and frequently interrupt it by PA to make it less harmful to overall health [11]. In addition, supervision and a motivational approach to rehabilitation after KA and HA have provided promising results [72-74], so the idea of a rehabilitation protocol incorporating high technology supporting a contemporary behavioral therapy approach seems reasonable preference in patients undergoing lower limb arthroplasty.

Because the study has several potential limitations, the results must be interpreted with caution. First, high heterogeneity was found in the analyses for some outcomes. This could be due to the fact that we comprehensively analyzed HA and KA, so high heterogeneity between the two subgroups could bias the results. Second, the data came from studies that used different activity monitors (bi-, uni- or triaxial accelerometers) with different reliability, validity and outcomes. Third, some authors have pointed out that measuring PA with objective data alone underestimates the realistic level of PA, so a combination of subjective and objective measurements is more appropriate when interpreting PA of patients after total joint arthroplasty [17]. Finally, we used the number of steps as a PA determinant, although it is not as well associated with general health as PA and SB [75]. We chose to do so because it has been most commonly used as a measure of PA and may help, at least in part, to explain the trajectories of PA in combination with MVPA and SB. To provide stronger evidence for practical implications, future studies examining PA and SB in patients after lower limb arthroplasty should use similar methodology and reliable instruments that provide objective data. The limitation of the current literature is that the studies either (a) compared patients and controls in single time points or (b) only tracked patients prospectively. Therefore, the progress of the patients with respect to the control groups is difficult to discern. 


\section{Conclusions}

The results suggest that objectively measured PA and functional performance increase while SB remain unchanged after lower limb arthroplasty. However, an optimal lifestyle is not achieved. Functional performance soon after surgery exceeds pre-operative levels and increases over time. In the period before and more than 12 months post-operation, patients tend to have lower functional capacity and are less physically active than their healthy comparison groups. Since the main goal of rehabilitation after lower limb arthroplasty is to improve patients' functional ability and general health, novel long-term rehabilitation approaches should be adapted to influence patients' lifestyle and in a way that maintains or even improves patients' overall health in the long term.

Supplementary Materials: The following are available online at https:/ /www.mdpi.com/article/10 $.3390 /$ jcm10245885/s1, Table S1: The study quality assessment.

Author Contributions: Conceptualization, N.Š., S.L., C.H., methodology, Ž.K.; software, Ž.K.; validation, N.Š., S.L.; formal analysis, M.S., Ž.K.; investigation, M.S.; resources, N.Š.; data curation, M.S., Ž.K.; writing—original draft preparation, M.S., Ž.K.; writing—review and editing, N.Š., C.H., S.L.; visualization, N.Š., C.H.; supervision, N.Š.; project administration, N.Š.; funding acquisition, N.Š., C.H., S.L. All authors have read and agreed to the published version of the manuscript.

Funding: We want to acknowledge the support of the European Regional Development Fund and Physiko- and Rheumatherapie Institute through the Centre of Active Ageing project in the Interreg Slovakia-Austria cross-border cooperation program (partners: Faculty for Physical Education and Sports, Comenius University in Bratislava: Institute for Physical Medicine and Rehabilitation, Physiko- and Rheumatherapie GmbH). Authors M.S., N.S. and Z.K. acknowledge the European Commission for funding the InnoRenew CoE project (Grant Agreement 739574) under the Horizon2020 Widespread-Teaming program. The funders had no role in study conceptualization, data acquisition, data analysis or manuscript preparation. The funders provided parts of authors salaries and will fund the submission fee if the paper is accepted.

Data Availability Statement: The data presented in this study are available on request from the corresponding author.

Conflicts of Interest: The authors declare no conflict of interest.

\section{References}

1. Picavet, H.S.J. Prevalence of self reported musculoskeletal diseases is high. Ann. Rheum. Dis. 2003, 62, 644-650. [CrossRef]

2. Smith, T.; Latham, S.; Maskrey, V.; Blyth, A. Patients' perceptions of physical activity before and after joint replacement: A systematic review with meta-ethnographic analysis. Postgrad. Med. J. 2015, 91, 483-491. [CrossRef]

3. Kurtz, S.M.; Ong, K.L.; Schmier, J.; Zhao, K.; Mowat, F.; Lau, E. Primary and Revision Arthroplasty Surgery Caseloads in the United States from 1990 to 2004. J. Arthroplast. 2009, 24, 195-203. [CrossRef]

4. Sadoghi, P.; Leithner, A.; Labek, G. Overcoming Boundaries of Worldwide Joint Arthroplasty Registers: The European Arthroplasty Register Minimal Dataset. J. Arthroplast. 2013, 28, 1327-1328. [CrossRef]

5. Nilsdotter, A.K.; Toksvig-Larsen, S.; Roos, E. Knee arthroplasty: Are patients' expectations fulfilled? Acta Orthop. 2009 , 80, 55-61. [CrossRef]

6. Paxton, R.J.; Melanson, E.L.; Stevens-Lapsley, J.E.; Christiansen, C.L. Physical activity after total knee arthroplasty: A critical review. World J. Orthop. 2015, 6, 614-622. [CrossRef] [PubMed]

7. Waldstein, W.; Kolbitsch, P.; Koller, U.; Boettner, F.; Windhager, R. Sport and physical activity following unicompartmental knee arthroplasty: A systematic review. Knee Surg. Sports Traumatol. Arthrosc. 2017, 25, 717-728. [CrossRef] [PubMed]

8. World Health Organization. Global Status Report on Noncommunicable Diseases; World Health Organization: Geneva, Switzerland, 2010; Volume 176.

9. Fiuza-Luces, C.; Garatachea, N.; Berger, N.A.; Lucia, A. Exercise is the Real Polypill. Physiology 2013, 28, 330-358. [CrossRef]

10. Pareja-Galeano, H.; Garatachea, N.; Lucia, A. Exercise as a Polypill for Chronic Diseases; Elsevier BV: Amsterdam, The Netherlands, 2015; Volume 135, pp. 497-526.

11. Bull, F.C.; Al-Ansari, S.S.; Biddle, S.; Borodulin, K.; Buman, M.P.; Cardon, G.; Carty, C.; Chaput, J.-P.; Chastin, S.; Chou, R.; et al. World Health Organization 2020 guidelines on physical activity and sedentary behaviour. Br. J. Sports Med. 2020, 54, 1451-1462. [CrossRef] [PubMed]

12. Koolhaas, C.M.; Dhana, K.; van Rooij, F.J.A.; Schoufour, J.D.; Hofman, A.; Franco, O. Physical activity types and health-related quality of life among middle-aged and elderly adults: The Rotterdam Study. J. Nutr. Health Aging 2018, 22, 246-253. [CrossRef] [PubMed] 
13. Beale, S.J.; Bending, M.W.; Trueman, P.; Naidoo, B. Should we invest in environmental interventions to encourage physical activity in England? An economic appraisal. Eur. J. Public Health 2012, 22, 869-873. [CrossRef]

14. Hagberg, L.A.; Lindholm, L. Review Article: Cost-effectiveness of healthcare-based interventions aimed at improving physical activity. Scand. J. Public Health 2006, 34, 641-653. [CrossRef]

15. Kastelic, K.; Šarabon, N. Comparison of Self-Reported Sedentary Time on Weekdays with an Objective Measure (activPAL). Meas. Phys. Educ. Exerc. Sci. 2019, 23, 227-236. [CrossRef]

16. Frimpong, E.; McVeigh, J.A.; Meiring, R.M. Sedentary Behavior in Patients With Knee Osteoarthritis Before and After Total Knee Arthroplasty: A Systematic Review. J. Aging Phys. Act. 2018, 26, 671-681. [CrossRef]

17. Mills, K.; Falchi, B.; Duckett, C.; Naylor, J. Minimal change in physical activity after lower limb joint arthroplasty, but the outcome measure may be contributing to the problem: A systematic review and meta-analysis. Physiotherapy 2019, 105, 35-45. [CrossRef]

18. Paxton, E.W.; Torres, A.; Love, R.M.; Barber, T.C.; Sheth, D.S.; Inacio, M. Total joint replacement: A multiple risk factor analysis of physical activity level 1-2 years postoperatively. Acta Orthop. 2016, 87, 44-49. [CrossRef]

19. Withers, T.M.; Lister, S.; Sackley, C.; Clark, A.; Smith, T.O. Is there a difference in physical activity levels in patients before and up to one year after unilateral total hip replacement? A systematic review and meta-analysis. Clin. Rehabil. 2016, 31, 639-650. [CrossRef] [PubMed]

20. Vissers, M.; Bussmann, J.; De Groot, I.; Verhaar, J.; Reijman, M. Walking and chair rising performed in the daily life situation before and after total hip arthroplasty. Osteoarthr. Cartil. 2011, 19, 1102-1107. [CrossRef]

21. Almeida, G.J.; Terhorst, L.; Irrgang, J.J.; Fitzgerald, G.K.; Jakicic, J.M.; Piva, S.R. Responsiveness of Physical Activity Measures Following Exercise Programs after Total Knee Arthroplasty. J. Exerc. Sports Orthop. 2017, 4, 1-8. [CrossRef] [PubMed]

22. WHO. More Active People for a Healthier World. J. Policy Model. 2018, 28, 615-627.

23. Page, M.J.; McKenzie, J.E.; Bossuyt, P.M.; Boutron, I.; Hoffmann, T.C.; Mulrow, C.D.; Shamseer, L.; Tetzlaff, J.M.; Akl, E.A.; Brennan, S.E.; et al. The PRISMA 2020 statement: An updated guideline for reporting systematic reviews. Int. J. Surg. 2021, 88, 105906. [CrossRef] [PubMed]

24. Higgins, J.; Green, S. Chapter 22: Overview of reviews. Cochrane handbook for systematic reviews of interventions. Cochrane Database Syst. Rev. 2008, 187-235.

25. Harbour, R.; Miller, J. A new system for grading recommendations in evidence based guidelines. BMJ 2001, 323, 7308. [CrossRef]

26. Wan, X.; Wang, W.; Liu, J.; Tong, T. Estimating the sample mean and standard deviation from the sample size, median, range and/or interquartile range. BMC Med. Res. Methodol. 2014, 14, 135. [CrossRef]

27. Moher, D.; Liberati, A.; Tetzlaff, J.; Altman, D.G.; The PRISMA Group. Ítems de referencia para publicar Revisiones Sistemáticas y Metaanálisis: La Declaración PRISMA. Rev. Española Nutr. Hum. Y Dietética 2014, 18, 172. [CrossRef]

28. Bade, M.J.; Kittelson, J.M.; Kohrt, W.M.; Stevens-Lapsley, J.E. Predicting Functional Performance and Range of Motion Outcomes After Total Knee Arthroplasty. Am. J. Phys. Med. Rehabil. 2014, 93, 579-585. [CrossRef]

29. Brandes, M.; Ringling, M.; Winter, C.; Hillmann, A.; Rosenbaum, D. Changes in physical activity and health-related quality of life during the first year after total knee arthroplasty. Arthritis Rheum. 2010, 63, 328-334. [CrossRef]

30. Caliskan, E.; Igdir, V.; Dogan, O.; Bicimoglu, A. Primary total knee replacement leads to an increase in physical activity but no changes in overall time of sedentary behaviour: A retrospective cohort study using an accelerometer. Int. Orthop. 2020, 44, 2597-2602. [CrossRef]

31. Casazza, G.A.; Lum, Z.C.; Giordani, M.; Meehan, J.P. Total Knee Arthroplasty: Fitness, Heart Disease Risk, and Quality of Life. J. Knee Surg. 2019, 33, 884-891. [CrossRef] [PubMed]

32. Cooper, N.A.; Rakel, B.A.; Zimmerman, M.B.; Tonelli, S.M.; Herr, K.A.; Clark, C.; Noiseux, N.O.; Callaghan, J.J.; Sluka, K. Predictors of multidimensional functional outcomes after total knee arthroplasty. J. Orthop. Res. 2017, 35, 2790-2798. [CrossRef]

33. Daugaard, R.; Tjur, M.; Sliepen, M.; Lipperts, M.; Grimm, B.; Mechlenburg, I. Are patients with knee osteoarthritis and patients with knee joint replacement as physically active as healthy persons? J. Orthop. Transl. 2018, 14, 8-15. [CrossRef] [PubMed]

34. Dayton, M.R.; Judd, D.L.; Hogan, C.A.; Stevens-Lapsley, J. Performance-Based Versus Self-Reported Outcomes Using the Hip Disability and Osteoarthritis Outcome Score After Total Hip Arthroplasty. Am. J. Phys. Med. Rehabil. 2016, 95, 132-138. [CrossRef] [PubMed]

35. De Groot, I.B.; Bussmann, H.J.; Stam, H.J.; Verhaar, J.A. Small Increase of Actual Physical Activity 6 Months After Total Hip or Knee Arthroplasty. Clin. Orthop. Relat. Res. 2008, 466, 2201-2208. [CrossRef]

36. Dominick, G.M.; Zeni, J.A.; White, D.K. Association of Psychosocial Factors With Physical Activity and Function After Total Knee Replacement: An Exploratory Study. Arch. Phys. Med. Rehabil. 2016, 97, S218-S225. [CrossRef]

37. Frimpong, E.; McVeigh, J.A.; Van Der Jagt, D.; Mokete, L.; Kaoje, Y.S.; Tikly, M.; Meiring, R.M. Light intensity physical activity increases and sedentary behavior decreases following total knee arthroplasty in patients with osteoarthritis. Knee Surg. Sports Traumatol. Arthrosc. 2018, 27, 2196-2205. [CrossRef] [PubMed]

38. Frimpong, E.; van der Jagt, D.R.; Mokete, L.; Pietrzak, J.; Kaoje, Y.S.; Smith, A.; McVeigh, J.A.; Meiring, R.M. Improvements in Objectively Measured Activity Behaviors Do Not Correlate with Improvements in Patient-Reported Outcome Measures Following Total Knee Arthroplasty. J. Arthroplast. 2020, 35, 712-719.e4. [CrossRef]

39. Fujita, K.; Makimoto, K.; Tanaka, R.; Mawatari, M.; Hotokebuchi, T. Prospective study of physical activity and quality of life in Japanese women undergoing total hip arthroplasty. J. Orthop. Sci. 2013, 18, 45-53. [CrossRef] [PubMed] 
40. Güler, T.; Sivas, F.; Yurdakul, F.G.; Çelen, E.; Utkan, A.; Başkan, B.; Bodur, H.; Özkurt, B. Early improvement in physical activity and function after total hip arthroplasty: Predictors of outcomes. Turk. J. Phys. Med. Rehabil. 2019, 65, 379-388. [CrossRef]

41. Hawke, L.J.; Shields, N.; Dowsey, M.M.; Choong, P.F.M.; Taylor, N.F. Physical activity levels after hip and knee joint replacement surgery: An observational study. Clin. Rheumatol. 2018, 38, 665-674. [CrossRef]

42. Heiberg, K.E.; Ekeland, A.; Bruun-Olsen, V.; Mengshoel, A.M. Recovery and Prediction of Physical Functioning Outcomes During the First Year After Total Hip Arthroplasty. Arch. Phys. Med. Rehabil. 2013, 94, 1352-1359. [CrossRef]

43. Höll, S.; Blum, A.; Gosheger, G.; Dieckmann, R.; Winter, C.; Rosenbaum, D. Clinical outcome and physical activity measured with StepWatch $3^{\mathrm{TM}}$ Activity Monitor after minimally invasive total hip arthroplasty. J. Orthop. Surg. Res. 2018, 13, 1-5. [CrossRef]

44. Jeldi, A.J.; Deakin, A.H.; Allen, D.J.; Granat, M.H.; Grant, M.; Stansfield, B.W. Total Hip Arthroplasty Improves Pain and Function but Not Physical Activity. J. Arthroplast. 2017, 32, 2191-2198. [CrossRef] [PubMed]

45. Kahn, T.L.; Schwarzkopf, R. Does Total Knee Arthroplasty Affect Physical Activity Levels? Data from the Osteoarthritis Initiative. J. Arthroplast. 2015, 30, 1521-1525. [CrossRef] [PubMed]

46. Ko, V.; Naylor, J.M.; Harris, I.A.; Crosbie, J.; Yeo, A.E. The six-minute walk test is an excellent predictor of functional ambulation after total knee arthroplasty. BMC Musculoskelet. Disord. 2013, 14, 145. [CrossRef] [PubMed]

47. Kuhn, M.; Harris-Hayes, M.; Steger-May, K.; Pashos, G.; Clohisy, J.C. Total Hip Arthroplasty in Patients 50 Years or Less. J. Arthroplast. 2013, 28, 872-876. [CrossRef] [PubMed]

48. Lin, B.A.; Thomas, P.; Spiezia, F.; Loppini, M.; Maffulli, N. Changes in daily physical activity before and after total hip arthroplasty. A pilot study using accelerometry. Surgeon 2013, 11, 87-91. [CrossRef] [PubMed]

49. Lützner, C.; Kirschner, S.; Lützner, J. Patient Activity After TKA Depends on Patient-specific Parameters. Clin. Orthop. Relat. Res. 2014, 472, 3933-3940. [CrossRef] [PubMed]

50. Lützner, C.; Beyer, F.; Kirschner, S.; Lützner, J. How Much Improvement in Patient Activity Can Be Expected After TKA? Orthopedics 2016, 39, S18-S23. [CrossRef] [PubMed]

51. Matsunaga-Myoji, Y.; Fujita, K.; Ide, S.; Tabuchi, Y.; Mawatari, M. Improved levels of physical activity in patients over 75 years following total knee arthroplasty. J. Orthop. Surg. 2019, 27, 1-5. [CrossRef]

52. Matsunaga-Myoji, Y.; Fujita, K.; Ide, S.; Tabuchi, Y.; Mawatari, M.; Makimoto, K. Changes in actual daily physical activity and patient-reported outcomes up to 2 years after total knee arthroplasty with arthritis. Geriatr. Nurs. 2020, 41, 949-955. [CrossRef] [PubMed]

53. Matsunaga-Myoji, Y.; Fujita, K.; Makimoto, K.; Tabuchi, Y.; Mawatari, M.; Matsunaga, Y. Three-Year Follow-Up Study of Physical Activity, Physical Function, and Health-Related Quality of Life After Total Hip Arthroplasty. J. Arthroplast. 2019, 35, 198-203. [CrossRef]

54. Moellenbeck, B.; Horst, F.; Gosheger, G.; Theil, C.; Seeber, L.; Kalisch, T. Sedentary Behavior in Older Patients before and after Total Hip Arthroplasty: A Prospective Cohort Study. Healthcare 2020, 8, 346. [CrossRef]

55. Moellenbeck, B.; Horst, F.; Gosheger, G.; Theil, C.; Seeber, L.; Kalisch, T. Does Total Hip or Knee Arthroplasty Have an Effect on the Patients' Functional or Behavioral Outcome and Health-Related Quality of Life of the Affected Partners? J. Arthroplast. 2021, 36, 885-891. [CrossRef]

56. Oka, T.; Ono, R.; Tsuboi, Y.; Wada, O.; Kaga, T.; Tamura, Y.; Yamamoto, Y.; Mizuno, K. Effect of preoperative sedentary behavior on clinical recovery after total knee arthroplasty: A prospective cohort study. Clin. Rheumatol. 2019, 39, 891-898. [CrossRef] [PubMed]

57. Rezzadeh, K.; Behery, O.A.; Kester, B.S.; Long, W.J.; Schwarzkopf, R. The Effect of Total Knee Arthroplasty on Physical Activity and Body Mass Index: An Analysis of the Osteoarthritis Initiative Cohort. Geriatr. Orthop. Surg. Rehabil. 2019, 10, 2151459318816480. [CrossRef] [PubMed]

58. Thewlis, D.; Bahl, J.S.; Fraysse, F.; Curness, K.; Arnold, J.; Taylor, M.; Callary, S.; Solomon, L. Objectively measured 24-hour activity profiles before and after total hip arthroplasty. Bone Jt. J. 2019, 101-B, 415-425. [CrossRef]

59. Tobinaga, T.; Obayashi, S.; Miyamoto, R.; Oba, K.; Abe, N.; Tsukamoto, S.; Ogawa, M.; Tochigi, Y.; Oka, K.; Ozeki, S. Factors influencing life-space mobility change after total knee arthroplasty in patients with severe knee osteoarthritis. J. Phys. Ther. Sci. 2019, 31, 889-894. [CrossRef] [PubMed]

60. Twiggs, J.; Salmon, L.; Kolos, E.; Bogue, E.; Miles, B.; Roe, J. Measurement of physical activity in the pre- and early post-operative period after total knee arthroplasty for Osteoarthritis using a Fitbit Flex device. Med. Eng. Phys. 2018, 51, 31-40. [CrossRef]

61. Vissers, M.; Bussmann, J.; de Groot, I.; Verhaar, J.; Reijman, M. Physical functioning four years after total hip and knee arthroplasty. Gait Posture 2013, 38, 310-315. [CrossRef]

62. Guedes, R.C.; Dias, J.M.D.; Dias, R.C.; Borges, V.S.; Lustosa, L.P.; Rosa, N.M.B. Total hip arthroplasty in the elderly: Impact on functional performance. Braz. J. Phys. Ther. 2011, 15, 123-130. [CrossRef]

63. De Luca, M.L.; Ciccarello, M.; Martorana, M.; Infantino, D.; Mauro, G.L.; Bonarelli, S.; Benedetti, M.G. Pain monitoring and management in a rehabilitation setting after total joint replacement. Medicine 2018, 97, e12484. [CrossRef]

64. Pinto, P.R.; McIntyre, T.; Araújo-Soares, V.; Almeida, A.; Ferrero, R. Persistent pain after total knee or hip arthroplasty: Differential study of prevalence, nature, and impact. J. Pain Res. 2013, 6, 691-703. [CrossRef] [PubMed]

65. Arnold, J.B.; Walters, J.L.; Ferrar, K. Does Physical Activity Increase After Total Hip or Knee Arthroplasty for Osteoarthritis? A Systematic Review. J. Orthop. Sports Phys. Ther. 2016, 46, 431-442. [CrossRef] [PubMed] 
66. Loyd, B.J.; Stackhouse, S.; Dayton, M.; Hogan, C.; Bade, M.; Stevens-Lapsley, J. The relationship between lower extremity swelling, quadriceps strength, and functional performance following total knee arthroplasty. Knee 2019, 26, 382-391. [CrossRef]

67. Vissers, M.M.; Bussmann, J.B.; Verhaar, J.; Arends, L.R.; Furlan, A.; Reijman, M. Recovery of Physical Functioning After Total Hip Arthroplasty: Systematic Review and Meta-Analysis of the Literature. Phys. Ther. 2011, 91, 615-629. [CrossRef]

68. Witjes, S.; Gouttebarge, V.; Kuijer, P.P.F.; van Geenen, R.; Poolman, R.; Kerkhoffs, G.M.M.J. Return to Sports and Physical Activity After Total and Unicondylar Knee Arthroplasty: A Systematic Review and Meta-Analysis. Sports Med. 2016, 46, 269-292. [CrossRef]

69. Cook, J.R.; Warren, M.; Ganley, K.J.; Prefontaine, P.; Wylie, J.W. A comprehensive joint replacement program for total knee arthroplasty: A descriptive study. BMC Musculoskelet. Disord. 2008, 9, 154. [CrossRef]

70. Hylkema, T.H.; Brouwer, S.; Kooijman, C.M.; De Vries, A.J.; Breukelman, F.; Dekker, H.; Almansa, J.; Kuijer, P.P.F.M.; Bulstra, S.K.; Stevens, M. Accelerometer Measured Sedentary and Physical Activity Behaviors of Working Patients after Total Knee Arthroplasty, and their Compensation Between Occupational and Leisure Time. J. Occup. Rehabil. 2021, 31, 350-359. [CrossRef]

71. Peter, W.F.; Nelissen, R.; Vlieland, T.V. Guideline Recommendations for Post-Acute Postoperative Physiotherapy in Total Hip and Knee Arthroplasty: Are They Used in Daily Clinical Practice? Musculoskelet. Care 2014, 12, 125-131. [CrossRef]

72. Christiansen, M.B.; Thoma, L.; Master, H.; Voinier, D.; Schmitt, L.A.; Ziegler, M.L.; LaValley, M.P.; White, D.K. Feasibility and Preliminary Outcomes of a Physical Therapist-Administered Physical Activity Intervention After Total Knee Replacement. Arthritis Rheum. 2020, 72, 661-668. [CrossRef] [PubMed]

73. Paxton, R.J.; Forster, J.E.; Miller, M.J.; Gerron, K.L.; Stevens-Lapsley, J.; Christiansen, C.L. A Feasibility Study for Improved Physical Activity After Total Knee Arthroplasty. J. Aging Phys. Act. 2018, 26, 7-13. [CrossRef] [PubMed]

74. Pellegrini, C.A.; Chang, R.W.; Dunlop, D.D.; Conroy, D.E.; Lee, J.; Van Horn, L.; Spring, B.; Cameron, K.A. Comparison of a Patient-Centered Weight Loss Program starting before versus after knee replacement: A pilot study. Obes. Res. Clin. Pract. 2018, 12, 472-478. [CrossRef] [PubMed]

75. White, D.K.; Tudor-Locke, C.; Felson, D.; Gross, K.D.; Niu, J.; Nevitt, M.; Lewis, C.E.; Torner, J.; Neogi, T. Walking to Meet Physical Activity Guidelines in Knee Osteoarthritis: Is 10,000 Steps Enough? Arch. Phys. Med. Rehabil. 2013, 94, 711-717. [CrossRef] [PubMed] 